\title{
A study of opacity in SOHO-SUMER and SOHO-CDS spectral observations
}

\section{The validity of the escape probability model}

\author{
G. A. Fischbacher, S. D. Loch, and H. P. Summers \\ University of Strathclyde, 107 Rottenrow, Glasgow G4 0NG, UK \\ Received 11 December 2001 / Accepted 15 April 2002

\begin{abstract}
The assumptions underpinning escape probability and absorption factor methods for describing spectral emission from optically thick plasmas, are addressed to test the range of validity of the associated expressions and to develop them for use within non-stratified atmospheres which include plasma flow and line blending. It is found that for moderate optical depths $\left(\tau_{0}<\sim 10\right)$ the escape probability is an effective tool for accurately describing the effect of opacity on emergent spectral line intensities. Furthermore it is found that the expressions may be used to extract optical depths directly from observational data independently of preconceived atmosphere model ideas. The analysis enables the detection of unresolved spicule-like structures at the solar limb.
\end{abstract}

Key words. atomic data - atomic processes - Sun: atmosphere - Sun: UV radiation

\section{Introduction}

The study of optically thick plasmas involves the solution of the coupled, non-linear equations of radiative transfer and statistical balance. Opacity couples each point in a plasma to each other point and thus knowledge of plasma conditions everywhere is necessary to model or interpret emission along a single line of sight. Optically thick emission may be modelled using radiative transfer techniques (Carlsson 1986; Olson et al. 1986), Monte Carlo simulations (Zheng et al. 1998; Code \& Whitney 1995; Guttebroze et al. 1986; Wood \& Raymond 2000) or escape probability methods (Holstein 1947; McWhirter 1965; Irons 1979; Hummer \& Rybicki 1982: Kastner \& Kastner 1990).

Radiative transfer methods solve the radiative transfer and statistical balance equations simultaneously and are effective in describing spectral line formation including partial frequency redistribution. However, due to their numerical demands, they are restricted to simple geometric configurations such as one dimensional (1D) plane parallel, semi-infinite slabs (e.g. Carlsson 1986; Heinzel et al. 1987; Lanzafame 1994; Hubeny \& Lites 1995; Goutikakis et al. 1997; Anzer \& Heinzel 1999, 2000) that are stratified with electron temperature and density based on

Send offprint requests to: H. P. Summers, e-mail: summers@phys.strath.ac.uk hydrostatic equilibrium (e.g. Vernazza et al. 1981, henceforth referred to as VAL), or 1D slabs that are vertically (Heinzel 1995) or horizontally (Mein et al. 1996) oriented and illuminated, perhaps, by an external source such as the underlying chromosphere and transition region. Two dimensional (2D) models have been developed which allow for transport in two directions but source geometries in such calculations remain relatively simple -2 D slabs (Paletou et al. 1993; Auer \& Paletou 1994; Paletou 1996) or isolated prominence threads (Fontenla et al. 1996) for example. In these models individual threads are described as plane-parallel slabs immersed in a radiation field due to the surrounding plasma. Astrophysical plasmas, such as those in the solar atmosphere, generally have a complex structure while radiative transfer diagnostics may only be interpreted within the simple geometries for which they apply.

Monte Carlo radiation transfer simulations naturally account for arbitrary illumination and multiple scattering in complex geometries. However, approximations are necessary and in this case there is a trade off between geometric complexity and detail in the line formation process. More specifically, these calculations model random walks of photons and so purely describe the scattering process. Many subtleties arising from absorption such as the influence of opacity on the source and contribution functions and ionisation balance are not included. 
Consequently these methods are restricted to moderate optical depths. This is not a severe restriction since many astronomical lines have low but non-zero optical depths. However, Monte Carlo techniques are model based and so, as with the radiative transfer techniques, they provide no means by which plasma parameters may be extracted directly from observations independently of a geometric model.

Escape probability methods rely on assumptions that simplify the source function term in the equations of radiative transfer and statistical balance. These assumptions decouple and linearise the equations. Consequently they provide approximate solutions to these equations in a moderate optical depth regime. This approach has the virtue of practicality. Escape probability expressions provide diagnostic tools to extract plasma parameters directly from observations. Furthermore they have the potential to be used in plasma models of arbitrary geometric complexity. For example, Orral \& Schmahl (1980) used attenuation factors (identical in principle to escape probabilities) to account for opacity within prominence models. These models consisted of resolved slabs or unresolved threads.

It was shown by Fischbacher et al. (2000 - hereafter referred to as Paper II) that the layer averaged escape probability $\left(\bar{g}\left\{\tau_{0}\right\}\right)$ is dependent purely on the line of sight optical depth. Thus it is independent of the density distribution along the line of sight. Therefore the plasma diagnostics based on this model may be interpreted independently of source configuration preconceptions.

We note that the validity of a solution to the radiative transfer and statistical balance equations (assuming any numerical schemes employed are precise) is entirely dependent on the quality of the assumptions made in prescribing the problem. It does not matter that a solution is numerically sophisticated if the underlying assumptions are invalid. This work considers the validity of the escape probability and absorption factor methods as valid tools for describing spectral emission from optically thick plasmas by assessing the assumptions that underpin the relevant expressions. This study will determine whether these techniques may be considered as accurate solutions or merely useful and insightful approximations.

It is found that for moderate optical depths $\left(\tau_{0}<\sim 10\right)$ the escape probability is an effective tool for accurately describing the effect of opacity on emergent spectral line intensities. The analysis also enables the detection of unresolved spicule-like structures at the solar limb.

\section{Summary of the escape probability/absorption factor approach}

The emission from an optically thick plasma is characterised by the equations of radiative transfer and statistical balance which may be written, following Paper II, as

$$
\frac{\mathrm{d} I_{u \rightarrow l}(s)}{\mathrm{d} s}=\frac{1}{4 \pi} A_{u \rightarrow l} N_{u}(s) g_{l u}(s)
$$

$$
\begin{aligned}
\frac{\mathrm{d} N_{u}(\boldsymbol{r})}{\mathrm{d} t}= & -A_{u \rightarrow l} N_{u}(\boldsymbol{r}) \Lambda_{l u}(\boldsymbol{r}) \\
& -\sum_{l^{\prime}<u, l^{\prime} \neq l} A_{u \rightarrow l^{\prime}} N_{u}(\boldsymbol{r}) \Lambda_{l^{\prime} u}(\boldsymbol{r}) \\
& +\sum_{u^{\prime}>u} A_{u^{\prime} \rightarrow u} N_{u^{\prime}}(\boldsymbol{r}) \Lambda_{u u^{\prime}}(\boldsymbol{r}) \\
& -\sum_{k} q_{u \rightarrow k} N_{\mathrm{e}} N_{u}(\boldsymbol{r})+\sum_{k} q_{k \rightarrow u} N_{\mathrm{e}} N_{k}(\boldsymbol{r})
\end{aligned}
$$

where

$$
\begin{aligned}
& g_{l u}(s)=\left[1-\frac{N_{l}(s) \omega_{u}}{N_{u}(s) \omega_{l}} \times \frac{c^{2}}{2 \nu_{0}^{2}} \int I_{\nu}(s) \phi_{\mathrm{e}}(\nu) \mathrm{d} \nu\right] \\
& \Lambda_{l u}(\boldsymbol{r})=\left[1-\frac{N_{l}(\boldsymbol{r}) \omega_{u}}{N_{u}(\boldsymbol{r}) \omega_{l}} \frac{c^{2}}{2 \nu_{0}^{2}} \times \int \bar{I}_{\nu}(\boldsymbol{r}) \phi_{\mathrm{a}}(\nu) \mathrm{d} \nu\right] .
\end{aligned}
$$

Here $d s$ is an element of distance along the line of sight, $A_{u \rightarrow l}$ is the Einstein $A$-coefficient for the $u \rightarrow l$ transition, $q_{u \rightarrow l}$ is the corresponding electron impact collisional rate coefficient with $N_{\mathrm{e}}$ the electron density, $N_{u}$ and $N_{l}$ are the upper and lower level population densities respectively, the $\omega$ 's are statistical weights and $\phi_{\mathrm{e}}(\nu)$ and $\phi_{\mathrm{a}}(\nu)$ are the emission and absorption profiles respectively. Statistical balance holds if all derivatives, $\mathrm{d} N_{u}(\boldsymbol{r}) / \mathrm{d} t$, are zero - that is, it holds for ions whose atomic populations are in steady state.

We focus on the $u \rightarrow l$ transition and omit the subscripts. Then $g(s)$ is the escape probability, representing the probability that a $u \rightarrow l$ photon emitted at the point $s$ in the direction of the line of sight, will escape the plasma. $\Lambda(\boldsymbol{r})$ is the Biberman-Holstein coefficient or netradiative bracket (Irons 1979) and is called here the $a b$ sorption factor following Paper II. It relates to the probability that a photo-absorption will occur at the point $\boldsymbol{r}$. Both terms may be viewed as providing parametric adjustments to the Einstein $A$-coefficients in the equations of radiative transfer and statistical balance. Thus, providing the $A$-coefficients are modified appropriately, the optically thick population structure may be obtained via an optically thin calculation.

If it is assumed that the source function $\left(S_{\nu}(\boldsymbol{r})\right.$, defined as the ratio of the emissivity (or emission coefficient), $j_{\nu}(\boldsymbol{r})$, to the opacity (or absorption coefficient), $\left.\kappa_{\nu}(\boldsymbol{r})\right)$ is constant in space then Eq. (1) becomes (see Paper II)

$I=\frac{1}{4 \pi} A_{u \rightarrow l} N_{u} \bar{g}\left\{\tau_{0}\right\} L$

where $L$ is the geometric length of the line of sight and $\bar{g}\left\{\tau_{0}\right\}$ is the line of sight averaged escape probability, which corresponds to the escape probability averaged along the line of sight and is given by (Kastner \& Kastner 1990; Brooks et al. 2000 - hereafter referred to as Paper I)

$\bar{g}\left\{\tau_{0}\right\}=\frac{1}{\sqrt{\pi}} \int_{-\infty}^{\infty}\left[\frac{1-\exp \left\{-\tau_{0} e^{-u^{2}}\right\}}{\tau_{0}}\right] \mathrm{d} u$. 
Note that since the source function is constant ( $\Rightarrow N_{u} / N_{l}=$ const. $)$, it follows that

$I=\left[\frac{2 \nu^{2}}{c^{2}} \frac{\omega_{l} N_{u}}{\omega_{u} N_{l}}\right] \tau_{0} \bar{g}\left\{\tau_{0}\right\} \sim \tau_{0} \bar{g}\left\{\tau_{0}\right\}$.

If the emitting layer is assumed to be a stratified semiinfinite slab of thickness $D$, with density varying only in the perpendicular direction ( $x$-axis), then the absorption factor may be written as

$\Lambda(\boldsymbol{r}) \equiv \mathcal{G}\left(\tau_{0}, x\right) \equiv \frac{1}{2}\left(\overline{\bar{g}}\left\{\tau_{0}^{-}\right\}+\overline{\bar{g}}\left\{\tau_{0}^{+}\right\}\right)$

where

$$
\begin{aligned}
\overline{\bar{g}}\left\{\tau_{0}\right\} & =\frac{1}{\sqrt{\pi}} \int_{-\infty}^{\infty} e^{-u^{2}}\left[\exp \left\{-\tau_{0} e^{-u^{2}}\right\}\right. \\
& \left.-\left\{\tau_{0} e^{-u^{2}}\right\} E_{1}\left\{\tau_{0} e^{-u^{2}}\right\}\right] \mathrm{d} u
\end{aligned}
$$

and

$$
\begin{gathered}
\tau_{0}^{-}=\tau_{0}(0, x) \equiv \int_{0}^{x} \kappa_{0}(x) \mathrm{d} x \\
\tau_{0}^{+}=\tau_{0}(x, L) \equiv \int_{x}^{D} \kappa_{0}(x) \mathrm{d} x
\end{gathered}
$$

$\overline{\bar{g}}\left\{\tau_{0}\right\}$ is identical to the function denoted by Bhatia \& Kastner (1997) as $\operatorname{SEFD}(C, T)$ (the slab escape factor, Doppler which is a function of position, $C$, in the layer and optical depth, $T$ - Bhatia \& Kastner's notation).

Optical depths may be deduced directly from spectral observations of branching ratios of lines arising from a common upper level via Eq. (5) (Jordan 1967; Doyle \& McWhirter 1980; Keenan \& Kingston 1986; Paper I).

\section{The effect of source function variation due to opacity}

\section{1. source}

The source function is dependent on position due to the spatial dependence of $N_{u} / N_{l}$. This ratio varies in space due to the variation of $\left(T_{\mathrm{e}}, N_{\mathrm{e}}\right)$, which leads to a spatially dependent population structure, and due to photoabsorption which enhances $N_{u}$ with respect to $N_{l}$.

If $T_{\mathrm{e}}$ and $N_{\mathrm{e}}$ are assumed to be constant such that the only spatial variation of the source function is due to opacity, then the effect of scattering into the line of sight may be considered. This phenomenon acts in opposition to the attenuation process along a line of sight. At points along the line of sight photons may be absorbed from any direction. If such absorption is followed by emission along the line of sight then there will be a positive contribution to the resultant emission.

This phenomena may be considered from two perspectives. Following Kastner \& Bhatia (1992), the intensity in a line may be written as

$I^{\text {thick }}=$ const. $\times \int_{\text {los }} N_{u}^{\text {thin }}(s) A_{u \rightarrow l}^{\text {thick }} g(s) \mathrm{d} s$.
Here the escape probability is modified to include scattering into the line of sight.

The second perspective may be summarised as

$I^{\text {thick }}=$ const. $\times \int_{\operatorname{los}} N_{u}^{\text {thick }}(s) A_{u \rightarrow l}^{\text {thin }} g(s) \mathrm{d} s$

and was compared with the approach of Bhatia \& Kastner (1999) in Paper II. Here the optically thick upper level population distribution along the line of sight $\left(N_{u}^{\text {thick }}(s)\right)$ is used and the only effect of opacity thereafter is to scatter photons out of the line of sight. These two perspectives were contrasted in Paper II.

$N_{u}^{\text {thick }}(s)$ may be obtained using the spatially resolved absorption factor which is given by

$$
\begin{aligned}
\Lambda\left(\tau_{0}, h\right)=1 & -\frac{1}{2 \sqrt{\pi}} \frac{N_{l}(h)}{N_{u}(h)} \int e^{-2 u^{2}}\left[\int_{0}^{h} N_{u}(x) \kappa_{0}\right. \\
& \times E_{1}\left\{e^{u^{2}} \tau_{0}(x, h)\right\} \mathrm{d} x \\
& \left.+\int_{h}^{D} N_{u}(x) \kappa_{0} E_{1}\left\{e^{u^{2}} \tau_{0}(h, x)\right\} \mathrm{d} x\right] \mathrm{d} \nu .
\end{aligned}
$$

The absorption factor, which has a functional dependence on the optical depth surface $\tau_{0}(x, h)$, is written here as $\Lambda\left(\tau_{0}, h\right)$ since it may be prescribed for a particular density distribution, $N_{l}(x)$, at particular position, $h$, by the disk centre total optical depth $\tau_{0}$. This is defined as

$\tau_{0}=\int_{0}^{D} \kappa_{0}(x) \mathrm{d} x$

The absorption factor calculation is iterative since $\Lambda\left(\tau_{0}, h\right)$ has a functional dependence on the upper level population density distribution, $N_{u}(x)$, which is opacity sensitive. Thus, the non-linearity of the statistical balance equations and their coupling with radiative transfer are restored. The iteration scheme is discussed in Paper II and it is an effective but not an efficient method. The present work does not seek to develop efficient ways of solving the equations of radiative transfer and statistical balance equations simultaneously but, rather, to determine the regime whereby these equations naturally linearise and decouple. In addition, this approach fits conveniently within the collisional radiative framework of the Atomic Data and Analysis Structure (ADAS - see Summers 2001) codes which are used here in the computations of statistical balance. Using $\Lambda\left(\tau_{0}, h\right)$ in a first order iteration scheme is effective up to some maximum optical depth which is larger than that for which the assumption of constant source function is valid. Therefore the approach here is satisfactory.

\subsection{The absorption factor calculation}

Figure 1 shows absorption factors versus position through an emitting layer with an exponentially varying density for the $2 \mathrm{~s}^{2} 2 \mathrm{p}^{2} \mathrm{P}_{3 / 2}-2 \mathrm{~s} 2 \mathrm{p}^{2}{ }^{2} \mathrm{P}_{3 / 2}$ and $2 \mathrm{~s}^{2} 2 \mathrm{p}^{2} \mathrm{P}_{1 / 2}-2 \mathrm{~s} 2 \mathrm{p}^{2}{ }^{2} \mathrm{P}_{3 / 2}$ lines of $\mathrm{C}$ II at $904.143 \AA$ and $903.620 \AA$ respectively. 


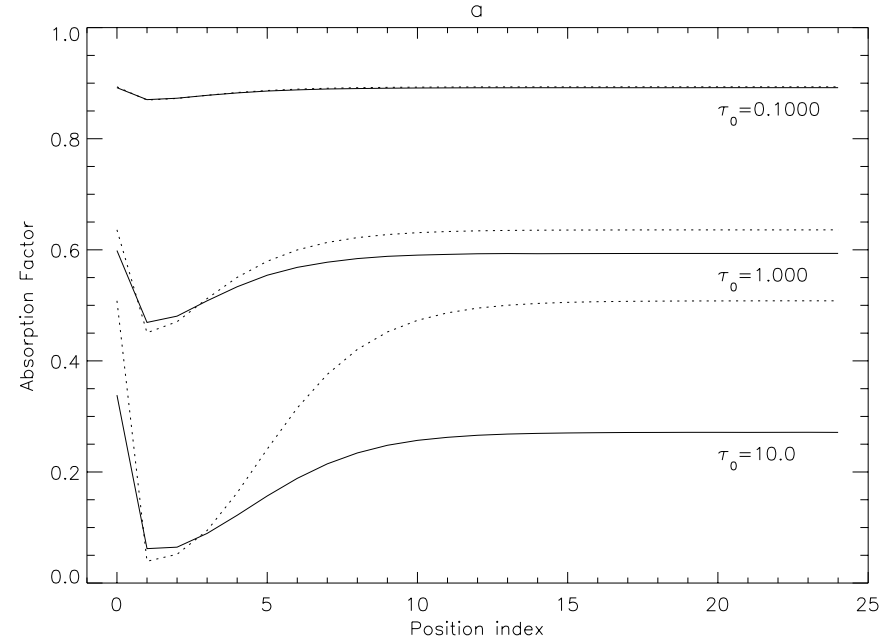

$b$

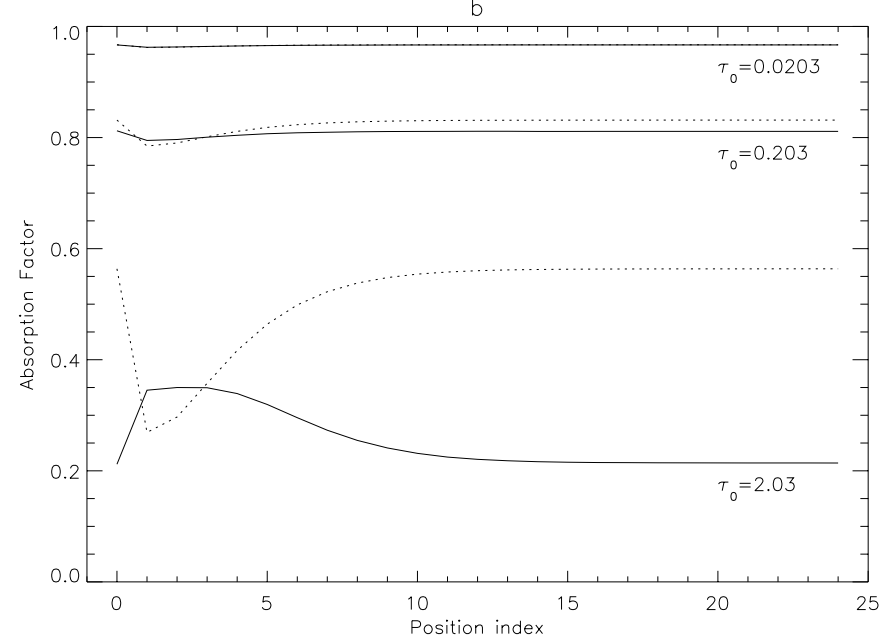

Fig. 1. Absorption factors versus position for the a) $2 \mathrm{~s}^{2} 2 \mathrm{p}{ }^{2} \mathrm{P}_{3 / 2}-2 \mathrm{~s} 2 \mathrm{p}^{2}{ }^{2} \mathrm{P}_{3 / 2}$ and b) $2 \mathrm{~s}^{2} 2 \mathrm{p}{ }^{2} \mathrm{P}_{1 / 2}-2 \mathrm{~s} 2 \mathrm{p}^{2}{ }^{2} \mathrm{P}_{3 / 2}$ lines of $\mathrm{C}$ II at $904.143 \AA$ and $903.620 \AA$ respectively. The solid lines correspond to $\Lambda\left(\tau_{0}, h\right)$ (Eq. (13)) and the dotted lines correspond to $\mathcal{G}\left(\tau_{0}, h\right)$ (Eq. (8)). The functional dependence of $\Lambda\left(\tau_{0}, h\right)$ on $N_{u}(x) / N_{u}(h)$, which is opacity sensitive, leads to the deviation of $\Lambda\left(\tau_{0}, h\right)$ from $\mathcal{G}\left(\tau_{0}, h\right)$. This deviation increases with optical depth and is most severe for lines that share an upper level with a line that is more optically thick as in case b). For such lines the $N_{u}(x) / N_{u}(h)$ curve is characterised by the optical depth of the thick partner rather than that of the line itself. The deviation is most severe at the layer edges where $R_{u}(x, h)>0$ for all $x$ (see Eq. (15)).

The curves are shown versus position index which relates to the computational grid and is equivalent to the geometric position in arbitrary units. The curves correspond to three pairs of optical depths, namely $(0.1,0.02),(1,0.2)$ and $(10,2)$. Considering firstly Fig. 1a, the $\Lambda\left(\tau_{0}, h\right)$ versus $x$ curve broadly follows that of $\mathcal{G}\left(\tau_{0}, h\right)$. Absorption is miminal at the layer edges where the plasma is exposed to a radiation field from only one side and thus the absorption factor is maximal here. This point is denoted $h_{\text {edge }}$. Absorption is at a maximum near the inner edge of the layer, close to the point of maximum density and thus the absorption factor is minimal here. This point is denoted $h_{\max }$. At this point the radiation field is maximal since the contribution from the points of maximum density are minimally attenuated, plus there is a contribution from the portion of the layer where the density is low.

Shown in Figs. 2a and b are absorption factors for $\mathrm{a}$ selection of C II lines, at heights $h_{\max }$ and $h_{\text {edge }}$ respectively. These reveal that absorption is greater within the layer than at the edge.

There is a secondary effect that acts in opposition to the above. Specifically, the variation in the degree of absorption as a function of space - i.e. the variation of $\Lambda\left(\tau_{0}, h\right)$ with $h$ - leads to a distortion of the upper level population density distribution. This is shown in Figs. 3a and $\mathrm{b}$ for the lines in Fig. 1 which share an upper level. The functional dependence that $\Lambda\left(\tau_{0}, h\right)$ has on the $N_{u}(x) / N_{u}(h)$ versus $x$ curve, which is opacity sensitive, is responsible for the deviation of $\Lambda\left(\tau_{0}, h\right)$ from $\mathcal{G}\left(\tau_{0}, h\right)$. If $R_{u}(x, h)$ is defined as

$R_{u}(x, h)=\frac{N_{u}^{\text {thick }}(x)}{N_{u}^{\text {thick }}(h)} \frac{N_{u}^{\text {thin }}(h)}{N_{u}^{\text {thin }}(x)}$

then $R_{u}\left(x, h_{\text {edge }}\right) \leq 1$ and $R_{u}\left(x, h_{\max }\right) \geq 1$ for all $x$. This follows from Fig. 3a. Consequently, the radiation field is enhanced at $h=h_{\text {edge }}$ and depleted at $h=h_{\max }$ in comparison to the $R_{u}(x, h)=$ const. case (i.e. as for $\mathcal{G}\left(\tau_{0}, h\right)$ ).

In Fig. 1b this secondary effect is dominant. For an optical depth of 2 the $\Lambda\left(\tau_{0}, h\right)$ versus $h$ trend is reversed with respect to that of $\mathcal{G}\left(\tau_{0}, h\right)$. Since this line shares its upper level with the $904.143 \AA$ line, which is more optically thick, its upper level is distorted much more than if it were the only thick line.

The applicability of $\mathcal{G}\left(\tau_{0}, h\right)$ therefore varies line-toline and with position. Figure 2 shows absorption factors versus optical depth at both $h_{\max }$ and $h_{\text {edge }}$. It is clear that agreement with $\mathcal{G}\left(\tau_{0}, h\right)$ is most pronounced at $h_{\max }$. At this point the indirect effects are minimal. At $h_{\text {edge }}$, however, they are significant.

It is clear, therefore, that the validity of $\mathcal{G}\left(\tau_{0}, h\right)$ in describing photo-absorption in a line is questionable. However, significant disagreement arises in lines that are relatively thin and thus also relatively weak. As such their significance in a statistical balance calculation is minimal. Consequently, though $\mathcal{G}\left(\tau_{0}, h\right)$ might be ineffective as an absorption factor for a particular line, its effectiveness in regard to calculating population structure distributions is determined by the strong lines, for which $\mathcal{G}\left(\tau_{0}, h\right)$ is more appropriate.

\subsection{The effect on the density distributions and emergent fluxes}

Figure 3a shows an opacity modified population density distribution for the $\mathrm{C}$ II $2 \mathrm{~s} 2 \mathrm{p}^{2} \quad{ }^{2} \mathrm{P}_{3 / 2}$ level for an optical depth of 10 in the $3 / 2-3 / 2$ line at $904.143 \AA$ (henceforth referred to as the control line). The deviation from the optically thin case is significant. 


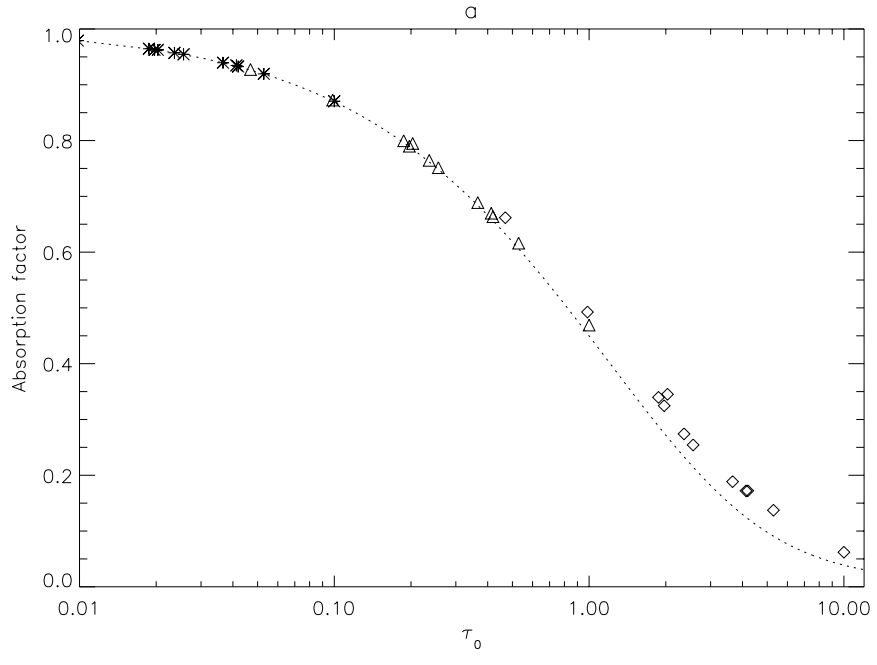

b

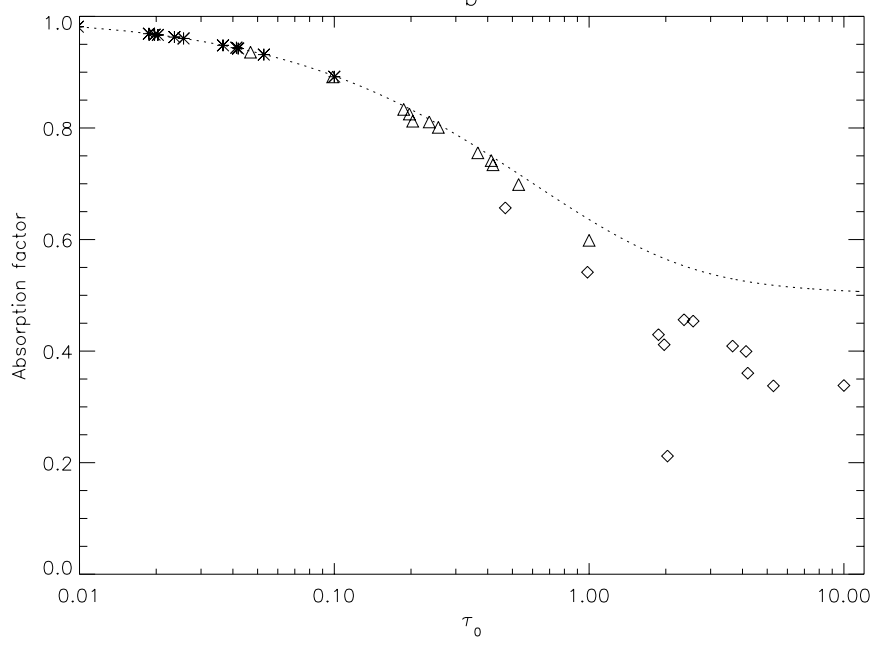

Fig. 2. Absorption factors versus optical depth at a) the point of maximum absorption in the $904.143 \AA$ line (position index $=$ 1.3 ) and $\mathbf{b}$ ) layer edge (position index $=0$ ), for selected lines of C II. The $*$ 's, $\triangle$ 's and $\diamond$ 's represent $\Lambda\left(\tau_{0}, h\right)$ values for three sets of optical depths corresponding to values of $0.1,1$ and 10 in the $904.143 \AA$ line (refered to as the control line). The dotted lines correspond to $\mathcal{G}\left(\tau_{0}, h_{\max }\right)$ and $\mathcal{G}\left(\tau_{0}, h_{\text {edge }}\right)$ in a) and $\mathbf{b})$ respectively. In a) $\mathcal{G}\left(\tau_{0}, h_{\max }\right)$ represents a minimum of the absorption factor since $R_{u}(x, h)<0$ for all $x$ (see Eq. (15)). In $\mathbf{b})$ the reverse is true.

In Fig. 3b $N_{u} / N_{l}$ population density ratios corresponding to the upper and lower levels of the control line, are plotted versus position index for optical depths of $0.1,1$ and 10 in the control line. These curves are indicative of the spatial variation of the source function which is modified in this case from constancy purely due to photoabsorption. From these it is evident that a regime exists in which the opacity is non-zero but $N_{u} / N_{l}$ is approximately constant. It is also clear that for an optical depth of 10 the modification is significant.

The upper level of the control line is also that of the C II $2 \mathrm{~s}^{2} 2 \mathrm{p}{ }^{2} \mathrm{P}_{1 / 2}-2 \mathrm{~s} 2 \mathrm{p}^{2}{ }^{2} \mathrm{P}_{3 / 2}$ line at $903.620 \AA$ which is less optically thick. Figure 3 a corresponds to an optical depth of 2 in this line and Fig. 3b corresponds to optical
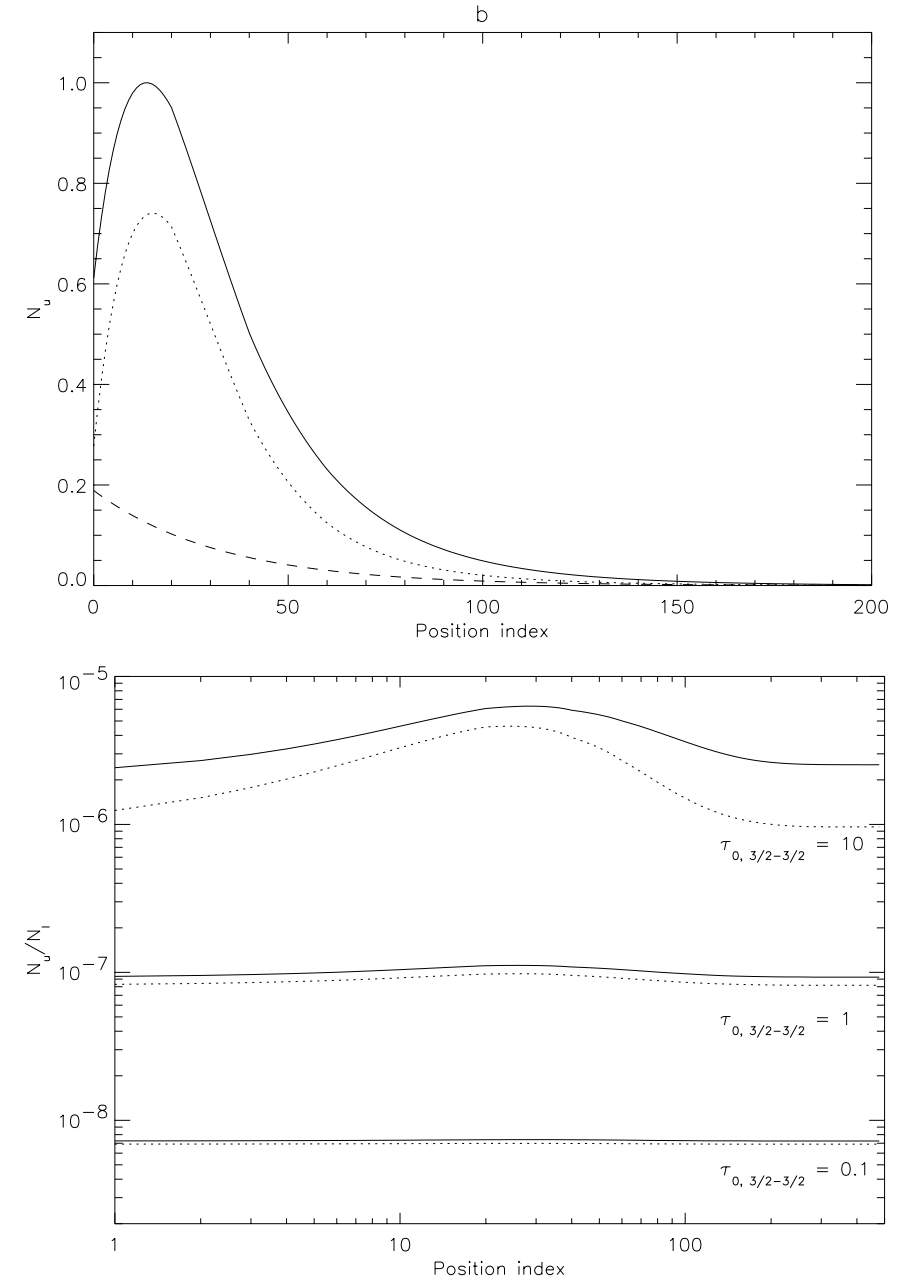

Fig. 3. a) Opacity modified population density distributions for the $\mathrm{C}$ II $2 \mathrm{~s} 2 \mathrm{p}^{2}{ }^{2} \mathrm{P}_{3 / 2}$ level for optical depths of 0 (dashed line) and 10 (solid and dotted lines) in the control line vs position index. b) $N\left(2 \mathrm{~s} 2 \mathrm{p}^{2}{ }^{2} \mathrm{P}_{3 / 2}\right) / N\left(2 \mathrm{~s}^{2} 2 \mathrm{p}^{2} \mathrm{P}_{3 / 2}\right)$ population density ratios for optical depths of $0.1,1$ and 10 in the control line plotted on a log-log scale. The solid lines corresponds to calculations based on $\Lambda\left(\tau_{0}, h\right)$ (Eq. (13)), and the dotted lines correspond to the $\mathcal{G}\left(\tau_{0}, h\right)$ based result (Eq. (8)).

depths of $0.02,0.2$ and 2 . Thus the distortion of the upper level of the $1 / 2-3 / 2$ line is significant for an optical depth of 2. It is for this reason that the absorption factor $\mathcal{G}\left(\tau_{0}, h\right)$ is restricted in its validity to $\tau_{0} \leq \sim 1$ at $h=h_{\max }$ and $\tau_{0} \leq \sim 0.5$ at $h=h_{\text {edge }}$.

The indirect effects upon the absorption factors evident in Figs. 1 and 2 (see sect. 3.2) are not present in the density distributions. Furthermore, from Fig. 4 it can be seen that for the emergent fluxes the indirect effects again are not evident. This figure shows predicted limb brightening curves for the $904.143 \AA$ (a) and $903.620 \AA$ (b) lines for the same set of optical depths as in Figs. 1 and 3b. In Fig. 4a the curves are calculated using Eq. (12), including the modified upper level population density distribution. They are close to those based on $\bar{g}\left\{\tau_{0}\right\}$ (using Eq. (5)) for $\tau_{0}=0.1,1$ and deviate most from one another for $\tau_{0}=10$. 

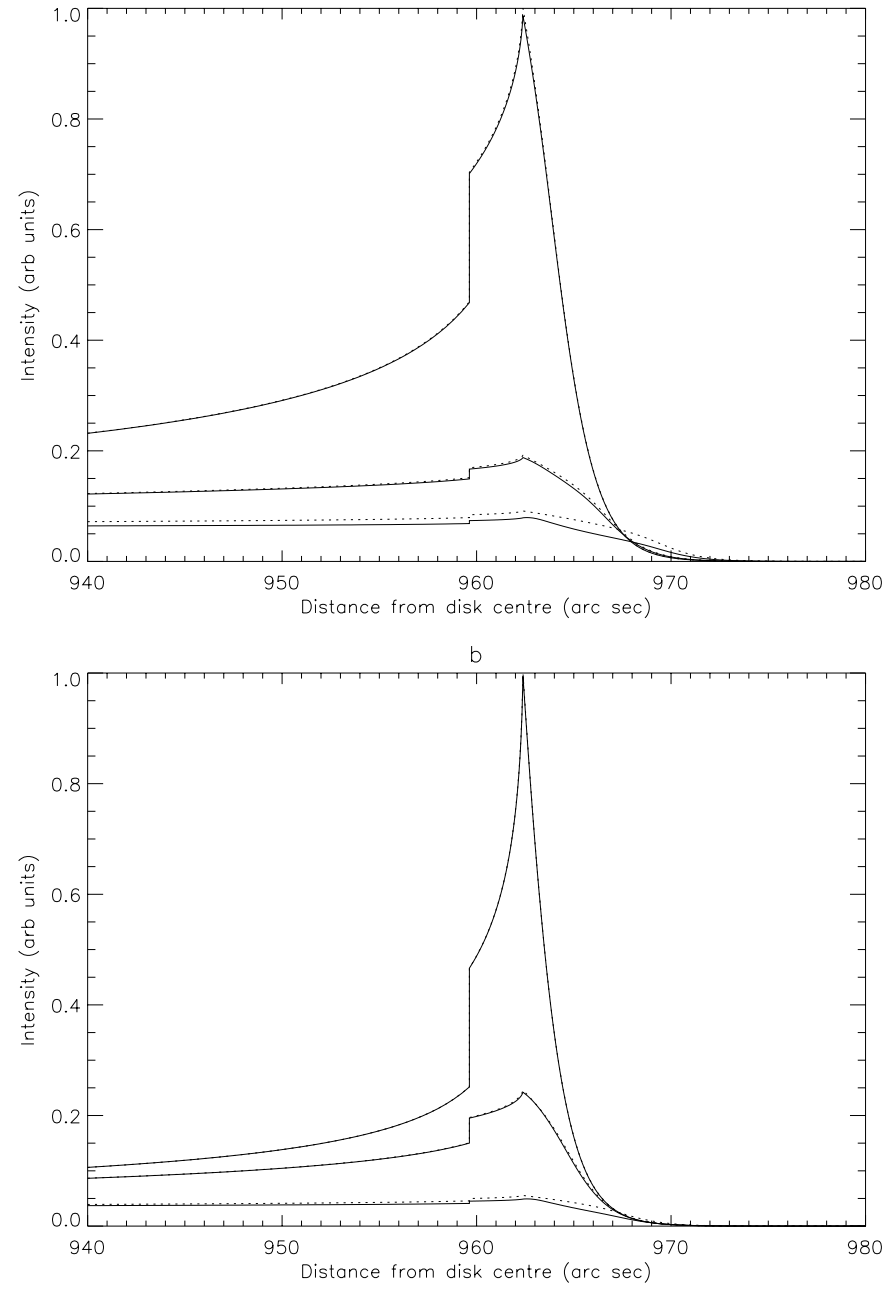

Fig. 4. Predicted limb brightening curves for a) the C II $2 \mathrm{~s}^{2} 2 \mathrm{p}^{2} \mathrm{P}_{3 / 2}-2 \mathrm{~s} 2 \mathrm{p}^{2}{ }^{2} \mathrm{P}_{3 / 2}$ line at $904.143 \AA$ for $\tau_{0}=0.1,1$ and 10 and $\mathbf{b}$ ) the $\mathrm{C}$ II $2 \mathrm{~s}^{2} 2 \mathrm{p}^{2} \mathrm{P}_{1 / 2}-2 \mathrm{~s} 2 \mathrm{p}^{2}{ }^{2} \mathrm{P}_{3 / 2}$ line at $903.620 \AA$ for $\tau_{0}=0.02,0.2$ and 2 . The solid lines correspond to calculations based in Eq. (12) which include an opacity modified upper level. The dotted lines correspond to those based on $\bar{g}\left\{\tau_{0}\right\}$ (using Eq. (5)).

\section{The effects of spectral line blending}

Spectral line blending was discussed in Paper II and can be included trivially, from an algebraic point of view, within the escape probability and absorption factor expressions. However, it re-introduces non-linearity into the statistical balance equation due to an explicit dependence on upper level population density ratios of overlapped components. These ratios are opacity sensitive and thus even $\mathcal{G}\left(\tau_{0}, h\right)$ (written in the blended case for line $i$ as $\mathcal{G}^{(i)}\left(\tau_{0}, h\right)$ ) must be calculated iteratively. The escape probabilities remain linear. The only effect of blending on them is to increase the effective line of sight optical depth as photons are absorbed by overlapped components. This introduces a dependence on lower level population density ratios which are insensitive to opacity.

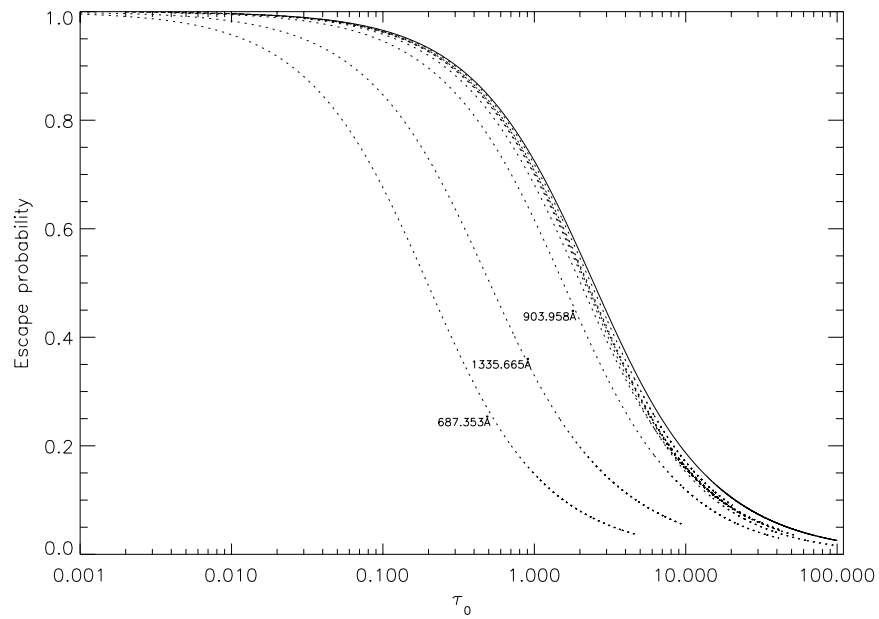

Fig. 5. line of sight averaged escape probabilities versus optical depth excluding line blending ( $\bar{g}\left\{\tau_{0}\right\}$ - solid line) and including line blending for a selection of spectral lines of $\mathrm{C}$ II $\left(\bar{g}^{(i)}\left\{\tau_{0}\right\}-\right.$ dotted lines). The three cases which deviate most markedly from the unblended result are labelled.

With blending included the line of sight averaged escape probability is given by (Paper II)

$$
\begin{aligned}
\bar{g}^{(i)}\left[\left\{\tau_{0}^{(n)}\right\},\right. & \left.\left\{v_{\text {in }}\right\}\right]=\frac{1}{\sqrt{\pi}} \int_{-\infty}^{\infty} e^{-u^{2} \times} \\
& {\left[\frac{1-\exp \left\{-\sum_{n} \tau_{0}^{(n)} e^{-\left(u+v_{i n}\right)^{2}}\right\}}{\sum_{n} \tau_{0}^{(n)} e^{-\left(u+v_{i n}\right)^{2}}}\right] \mathrm{d} u }
\end{aligned}
$$

where

$v_{i n}=\frac{\nu_{0}^{(i)}-\nu_{0}^{(n)}}{\Delta \nu_{D}}$

$\bar{g}^{(i)}\left[\left\{\tau_{0}^{(n)}\right\},\left\{v_{i n}\right\}\right]$ is prescribed by a set of optical depths, $\left\{\tau_{0}^{(n)}\right\}$ and a set of overlap parameters $\left\{v_{i n}\right\}$. The latter are constants, though they may act as variable parameters in an emission model or diagnostic. Furthermore, the set of optical depths may be related via $\tau_{0}^{(i)}=$ const. $\times \tau_{0}^{(n)}$ (see Paper I) and so the escape probabilities become prescribed by a single optical depth and a set of optical depth ratios, $\left\{\tau_{0}^{(n)} / \tau_{0}^{i}\right\}$. Thus $\bar{g}^{(i)}\left[\left\{\tau_{0}^{(n)}\right\},\left\{v_{i n}\right\}\right]$ becomes $\bar{g}^{(i)}\left\{\tau_{0}\right\}$ and may be plotted for line $i$ as a function of optical depth. Blended escape probabilities are shown in Fig. 5. It can be seen that line blending always increases the the absorption along the line of sight ${ }^{1}$ and therefore decreases the escape probability. The unblended quantity therefore represents an upper limit. A corresponding expression exists for the escape probability which is written for line $i$ as $g^{(i)}(s)$.

A further effect of line blending is to produce an asymmetry in the emergent spectral line profiles. This occurs since overlapped lines are generally displaced in frequency

1 This is evident in Tables 1 and 2 where smaller optical depths are required in the blended case to produce the same opacity effect as in the unblended case. 
space from one another. The emergent line profiles may be examined from Eq. (16) since

$$
\begin{aligned}
I_{\nu} \sim \bar{N}_{u} \frac{1}{\sqrt{\pi}} e^{-u^{2}} \times \\
\left.\qquad \frac{1-\exp \left\{-\sum_{n} \tau_{0}^{(n)} e^{-\left(u+v_{i n}\right)^{2}}\right\}}{\sum_{n} \tau_{0}^{(n)} e^{-\left(u+v_{i n}\right)^{2}}}\right] .
\end{aligned}
$$

If blending is neglected Eq. (18) is the same as that used by Doyle et al. (2000) to study the effects of line broadening due to opacity in spectral lines from the solar transition region.

The blended escape probabilities and absorption factors and opacity modified line profiles were discussed in more detail in Paper II.

Using the blended escape probability expression, optical depths may be directly extracted from observations of branching ratios of lines arising from a common upper level, as described in Paper I. Results for C III $2 \mathrm{~s} 2 \mathrm{p}^{3} \mathrm{P}_{2}-2 \mathrm{p}^{2}{ }^{3} \mathrm{P}_{2}$, C II $2 \mathrm{~s}^{2} 2 \mathrm{p}^{2} \mathrm{P}_{3 / 2}-2 \mathrm{~s} 2 \mathrm{p}^{2}{ }^{2} \mathrm{P}_{3 / 2}$ and $\mathrm{C}$ II $2 \mathrm{~s}^{2} 2 \mathrm{p}^{2} \mathrm{P}_{3 / 2}-2 \mathrm{~s} 2 \mathrm{p}^{2}{ }^{2} \mathrm{~S}_{1 / 2}$ are shown in Tables $1-3$ respectively. Corresponding values in the unblended case are shown in brackets. These differ slightly from those in Paper I due to inaccuracies in the escape probability calculation in that work. In the C III $2 \mathrm{~s} 2 \mathrm{p}^{3} \mathrm{P}_{2}-2 \mathrm{p}^{2}{ }^{3} \mathrm{P}_{2}$ case optical depths cannot be extracted at 958.06 and 959.94 arcsec if blending is excluded. Blending increases the effective optical depth ratio causing the intensity ratio to vary more strongly with optical depth. In the C II $2 \mathrm{~s}^{2} 2 \mathrm{p}{ }^{2} \mathrm{P}_{3 / 2}-2 \mathrm{~s} 2 \mathrm{p}^{2}{ }^{2} \mathrm{~S}_{1 / 2}$ case optical depths cannot be extracted at heights of 972.78 arcsec and above due to uncertainties in the fit at these positions.

There are two effects upon the absorption factors. Firstly there is the increase in effective optical depth as in the escape probability case. Secondly there is a nonlinear effect due to the dependence on upper level population density ratios. The former enhances the degree of absorption and acts identically to the unblended case but with an increased optical depth. The latter, however, can lead to an enhancement or depletion of absorption (though there is always a net enhancement when both effects are accounted for) which is approximately constant in space. The resultant effect is a shift in the absorption factors, upward or downward with respect to the $\mathcal{G}^{(i)}\left(\tau_{0}, h\right)$ values. This is illustrated in Figs. 6 and 7 .

Blended absorption factors are shown in Fig. 7 which contrasts the $\Lambda^{(i)}\left(\tau_{0}, h\right)$ with the $\mathcal{G}^{(i)}\left(\tau_{0}, h\right)$ values. As in the unblended case agreement is more pronounced when $h=h_{\max }$ and the discrepency is greatest at layer edge $\left(h=h_{\text {edge }}\right)$. It can also be seen that for the most severely blended lines the deviation between the two quantities is more significant than for the less significantly blended components.

The non-linear influence of the upper level population density dependence impinges upon calculability of the absorption factor and on its absolute value but does not
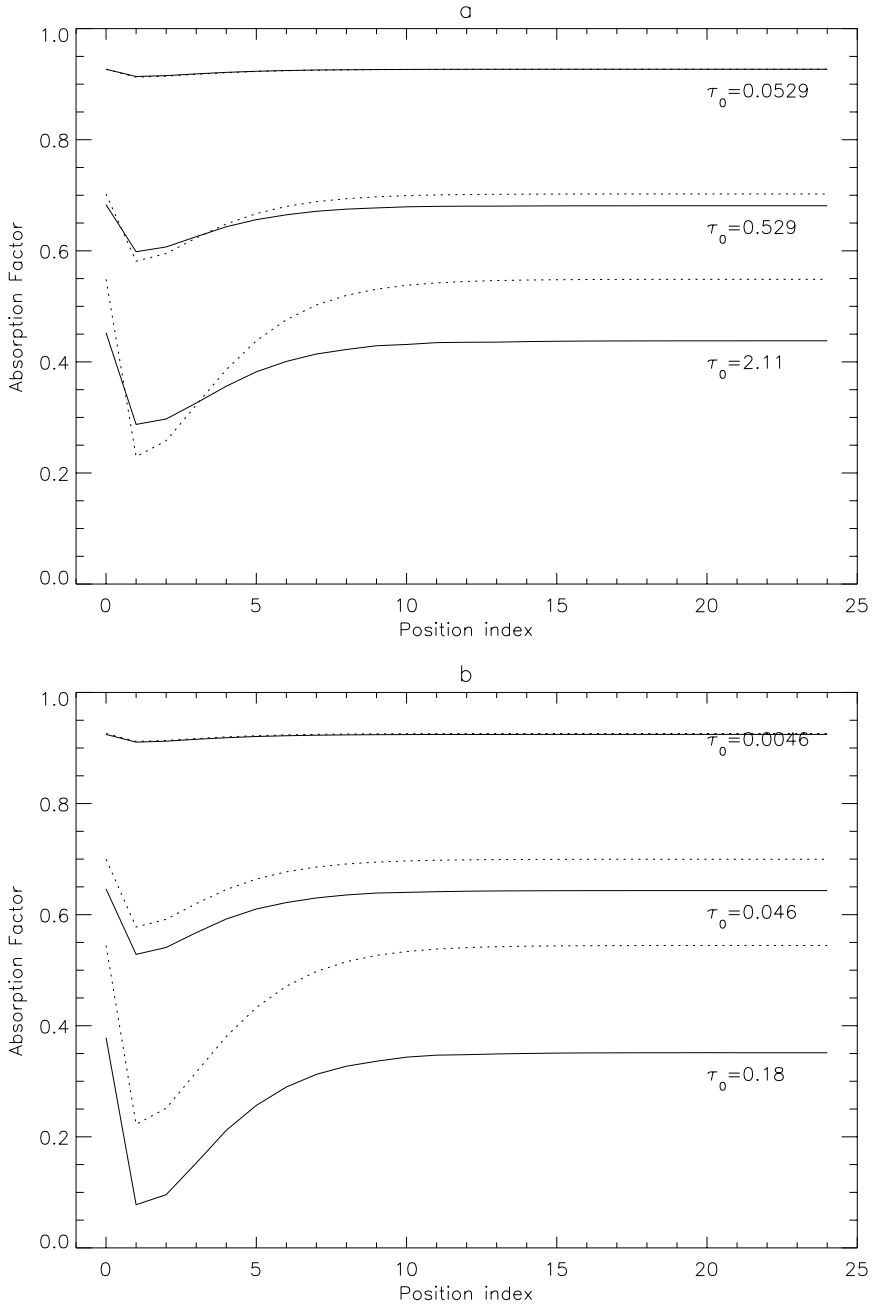

Fig. 6. Absorption factors versus position for the a) $2 s^{2} 2 p$ ${ }^{2} \mathrm{P}_{3 / 2}-2 \mathrm{~s}^{2} 3 \mathrm{~d}^{2} \mathrm{D}_{5 / 2}$ and b) $2 \mathrm{~s}^{2} 2 \mathrm{p}^{2} \mathrm{P}_{3 / 2}-2 \mathrm{~s}^{2} 3 \mathrm{~d}^{2} \mathrm{D}_{3 / 2}$ lines of $\mathrm{C}$ II at $687.346 \AA$ and $687.353 \AA$ respectively. The solid lines correspond to $\Lambda^{(i)}\left(\tau_{0}, h\right)$ and the dotted lines correspond to $\mathcal{G}^{(i)}\left(\tau_{0}, h\right)$. As well as sharing an upper level with the $1 / 2$ $3 / 2$ component at $687.051 \AA$, the $3 / 2-3 / 2$ line $(687.353 \AA)$ is blended with the $3 / 2-5 / 2$ one $(687.346 \AA)$. Since it is significantly weaker than the blended component, the $3 / 2-3 / 2$ line is markedly modified due to absorption of $3 / 2-3 / 2$ photons in the $3 / 2-5 / 2$ line. This effect is only weakly dependent on spatial position and so leads to no significant distortion of the $\Lambda^{(i)}\left(\tau_{0}, h\right)$ versus $\mathrm{h}$ curve but, rather, leads to a constant downward shift.

significantly alter the absorption factor versus position distributions. Thus it is purely the increase in effective optical depth that affects the validity of the line of sight averaged escape probability.

Figure 7 shows blended absorption factors for selected lines of C II, plotted against optical depth. As in the unblended case, agreement between the $\Lambda^{(i)}\left(\tau_{0}, h\right)$ and the $\mathcal{G}^{(i)}\left(\tau_{0}, h\right)$ values is more pronounced when $h=h_{\max }$ than at layer edge and at all positions in the layer, the agreement decreases with optical depth. The discrepancy between $\Lambda^{(i)}\left(\tau_{0}, h\right)$ and $\mathcal{G}^{(i)}\left(\tau_{0}, h\right)$ is due to both the modification to the upper level population distributions, which 
Table 1. Optical depths and corresponding escape probabilities for the C III $2 \mathrm{~s} 2 \mathrm{p}{ }^{3} \mathrm{P}_{2}-2 \mathrm{p}^{2}{ }^{3} \mathrm{P}_{2}(1175.711 \AA)$ transition extracted from branching ratios of the 2-2 line over the 1-2 line of the $2 \mathrm{~s} 2 \mathrm{p}{ }^{3} \mathrm{P}-2 \mathrm{p}^{2}{ }^{3} \mathrm{P}$ multiplet, observed by SUMER (see Paper I). Values in brackets correspond to unblended calculations. This table corresponds to Table 3 of Paper I. The pointing positions are adjusted here by 3.4 arc sec with respect to those in Paper I to account for the pointing error found therein.

\begin{tabular}{llllll}
\hline \hline Pos. $\left({ }^{\prime \prime}\right)$ & $\tau_{0,2-2}$ & $\bar{g}\left\{\tau_{0,2-2}\right\}$ & Pos. $\left({ }^{\prime \prime}\right)$ & $\tau_{0,2-2}$ & $\bar{g}\left\{\tau_{0,2-2}\right\}$ \\
\hline 946.46 & $0.61(0.74)$ & $0.80(0.78)$ & 963.34 & $54.0(-)$ & $0.03(-)$ \\
948.34 & $0.80(0.94)$ & $0.75(0.74)$ & 965.28 & $5.40(8.64)$ & $0.27(0.21)$ \\
950.21 & $0.90(1.09)$ & $0.73(0.71)$ & 967.15 & $1.40(1.68)$ & $0.62(0.60)$ \\
952.09 & $1.05(1.27)$ & $0.69(0.67)$ & 969.03 & $1.04(1.27)$ & $0.70(0.67)$ \\
953.96 & $0.86(1.05)$ & $0.74(0.71)$ & 970.90 & $1.20(1.42)$ & $0.66(0.64)$ \\
955.84 & $1.00(1.18)$ & $0.71(0.69)$ & 972.78 & $1.50(1.81)$ & $0.61(0.58)$ \\
957.71 & $1.51(1.86)$ & $0.60(0.58)$ & 974.65 & $1.40(1.67)$ & $0.62(0.60)$ \\
959.59 & $2.70(3.42)$ & $0.45(0.41)$ & 976.53 & $1.81(2.24)$ & $0.56(0.53)$ \\
961.46 & $15.0(-)$ & $0.11(-)$ & 978.40 & $1.72(2.13)$ & $0.57(0.54)$ \\
\hline
\end{tabular}

Table 2. Optical depths and corresponding escape probabilities for the C II $2 \mathrm{~s}^{2} 2 \mathrm{p}^{2} \mathrm{P}_{3 / 2}-2 \mathrm{~s} 2 \mathrm{p}^{2}{ }^{2} \mathrm{P}_{3 / 2}(904.143 \AA)$ transition extracted from branching ratios of the $3 / 2-3 / 2$ line over the $1 / 2-3 / 2$ line of the $2 \mathrm{~s}^{2} 2 \mathrm{p}^{2} \mathrm{P}-2 \mathrm{~s} 2 \mathrm{p}^{2}{ }^{2} \mathrm{P}$ multiplet, observed by SUMER (see Paper I). Values in brackets correspond to unblended calculations. This table corresponds to Table 7 of Paper I.

\begin{tabular}{llllll}
\hline \hline Pos. $\left(^{\prime \prime}\right)$ & $\tau_{0,3 / 2-3 / 2}$ & $\bar{g}\left\{\tau_{0,3 / 2-3 / 2}\right\}$ & Pos. $\left(^{\prime \prime}\right)$ & $\tau_{0,3 / 2-3 / 2}$ & $\bar{g}\left\{\tau_{0,3 / 2-3 / 2}\right\}$ \\
\hline 946.46 & $3.20(4.95)$ & $0.39(0.32)$ & 963.34 & $3.05(4.69)$ & $0.40(0.33)$ \\
948.34 & $3.23(5.01)$ & $0.39(0.32)$ & 965.28 & $3.13(4.84)$ & $0.40(0.33)$ \\
950.21 & $2.71(4.13)$ & $0.43(0.36)$ & 967.15 & $2.90(4.40)$ & $0.42(0.35)$ \\
952.09 & $2.86(4.37)$ & $0.42(0.35)$ & 969.03 & $1.90(2.88)$ & $0.53(0.46)$ \\
953.96 & $3.31(5.15)$ & $0.38(0.31)$ & 970.90 & $1.54(2.36)$ & $0.59(0.51)$ \\
955.84 & $3.12(4.81)$ & $0.40(0.33)$ & 972.78 & $0.58(1.07)$ & $0.81(0.71)$ \\
957.71 & $3.00(4.56)$ & $0.41(0.34)$ & 974.65 & $0.83(1.40)$ & $0.74(0.65)$ \\
959.59 & $3.40(5.23)$ & $0.37(0.31)$ & 976.53 & $1.01(1.63)$ & $0.70(0.61)$ \\
961.46 & $3.30(5.10)$ & $0.38(0.31)$ & 978.40 & $0.75(1.29)$ & $0.76(0.67)$ \\
\hline
\end{tabular}

Table 3. Optical depths and corresponding escape probabilities for the C II $2 \mathrm{~s}^{2} 2 \mathrm{p}^{2} \mathrm{P}_{3 / 2}-2 \mathrm{~s} 2 \mathrm{p}^{2}{ }^{2} \mathrm{~S}_{1 / 2}(1037.012 \AA)$ transition extracted from branching ratios of the $3 / 2-1 / 2$ line over the $1 / 2-1 / 2$ line of the $2 \mathrm{~s}^{2} 2 \mathrm{p}^{2} \mathrm{P}-2 \mathrm{~s} 2 \mathrm{p}^{2}{ }^{2} \mathrm{~S}$ multiplet, observed by SUMER (see Paper I). Values in brackets correspond to unblended calculations. This table corresponds to Table 5 of Paper I. The blended and unblended values are the same since since there is no significant overlap in these lines.

\begin{tabular}{llllll}
\hline \hline Pos. $\left({ }^{\prime \prime}\right)$ & $\tau_{0,3 / 2-1 / 2}$ & $\bar{g}\left\{\tau_{0,3 / 2-1 / 2}\right\}$ & Pos. $\left(^{\prime \prime}\right)$ & $\tau_{0,3 / 2-1 / 2}$ & $\bar{g}\left\{\tau_{0,3 / 2-1 / 2}\right\}$ \\
\hline 946.46 & $5.30(5.30)$ & $0.31(0.31)$ & 963.34 & $10.2(10.2)$ & $0.18(0.18)$ \\
948.34 & $5.25(5.25)$ & $0.31(0.31)$ & 965.28 & $6.00(6.00)$ & $0.28(0.28)$ \\
950.21 & $4.95(4.95)$ & $0.32(0.32)$ & 967.15 & $2.82(2.82)$ & $0.46(0.46)$ \\
952.09 & $5.70(5.70)$ & $0.29(0.29)$ & 969.03 & $0.36(0.36)$ & $0.88(0.88)$ \\
953.96 & $6.23(6.23)$ & $0.27(0.27)$ & 970.90 & $0.36(0.36)$ & $0.88(0.88)$ \\
955.84 & $6.00(6.00)$ & $0.28(0.28)$ & 972.78 & $-(-)$ & $-(-)$ \\
957.71 & $6.70(6.70)$ & $0.26(0.26)$ & 974.65 & $-(-)$ & $-(-)$ \\
959.59 & $5.80(5.80)$ & $0.29(0.29)$ & 976.53 & $-(-)$ & $-(-)$ \\
961.46 & $6.20(6.20)$ & $0.27(0.27)$ & 978.40 & $-(-)$ & $-(-)$ \\
\hline
\end{tabular}

$\mathcal{G}^{(i)}\left(\tau_{0}, h\right)$ neglects, and the upper level population density ratios which are also modified at each point. If $\mathcal{G}^{(i)}\left(\tau_{0}, h\right)$ is calculated iteratively then opacity modified upper level population density ratios are obtained and the discrepancy between $\Lambda^{(i)}\left(\tau_{0}, h\right)$ and $\mathcal{G}^{(i)}\left(\tau_{0}, h\right)$ is solely due to the modification to the upper level population distributions, as in the unblended case, and thus the agreement between the two is enhanced. Then the only influence of blending is to increase the effective optical depth.
Figure 8a shows limb-brightening curves for the control line (at 904.143 $\AA$ ) including (solid line) and excluding (dotted line) the modification to the population structure. As in the unblended case, for low optical depths the population modification has a negligible influence on the emission curves but becomes significant as the optical depth increases. However, the maximum optical depth for which the $\bar{g}^{(i)}\left\{\tau_{0}\right\}$ is effective is reduced in comparison with the unblended case. For a disk centre optical depth of 4 , there 

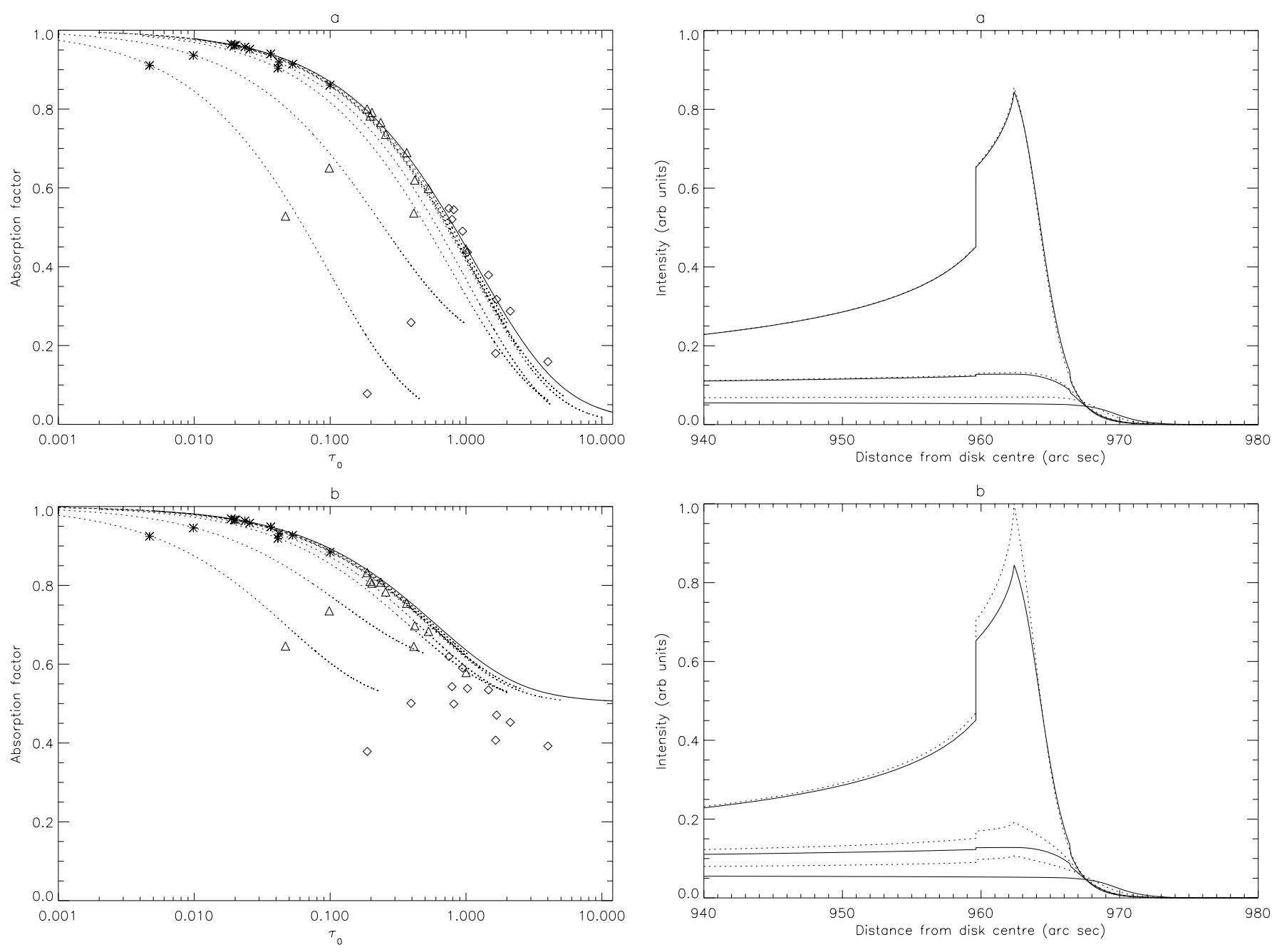

Fig. 7. Absorption factors including line blending versus optical depth at a) the point of maximum absorption in the control line (position index $=1$ ) and $\mathbf{b}$ ) layer edge (position index = $0)$, for selected lines of C II. The $*$ 's, $\triangle$ 's and $\diamond$ 's represent $\Lambda^{(i)}\left(\tau_{0}, h\right)$ values for three sets of optical depths corresponding to values of $0.1,1$ and 4 in the control line. The dotted lines correspond to $\mathcal{G}^{(i)}\left(\tau_{0}, h_{\max }\right)$ and $\mathcal{G}^{(i)}\left(\tau_{0}, h_{\text {edge }}\right)$ in a) and $\left.\mathbf{b}\right)$ respectively.

is a noticable deviation between the $\bar{g}^{(i)}\left\{\tau_{0}\right\}$ and $g^{(i)}(s)$ based models. Nevertheless, it is clear that a regime exists within which the effects of opacity are significant and $\bar{g}^{(i)}\left\{\tau_{0}\right\}$ is effective.

Figure 8b shows limb-brightening curves for the control line excluding (solid line) and including (dotted line) line blending. The difference is marked even for a disk centre optical depth of 0.1. Thus line blending cannot be neglected even for small optical depths. Figures $8 \mathrm{a}$ and $\mathrm{b}$ demonstrate that the inclusion of line blending in the escape probability expression is a more significant correction than the inclusion of an opacity modified upper level population distribution. However, the region of validity of the escape probability is still determined by the extent of the population modification.

Fig. 8. Predicted limb brightening curves for the $\mathrm{C}$ II $2 \mathrm{~s}^{2} 2 \mathrm{p}{ }^{2} \mathrm{P}_{3 / 2}-2 \mathrm{~s} 2 \mathrm{p}^{2}{ }^{2} \mathrm{P}_{3 / 2}$ line at $904.143 \AA$ for $\tau=$ 0.1, 1 and 4. In both $\mathbf{a}$ ) and $\mathbf{b}$ ) the solid lines correspond to calculations based on Eq. (12) which include both line blending and an opacity modified upper level. In a), the dotted lines correspond to those with the population modification excluded and blending included (via Eq. (16)). In b), the dotted lines correspond to those based on $\bar{g}\left\{\tau_{0}\right\}$ (using Eq. (6)) which neglect both the modification to the populations and line blending. It is clear that line blending has a more marked influence on the limb-brightening curves.

\section{Non-stratified models}

The atmosphere models examined in Papers I and II are simple but the only structural restriction on them, as far as the escape probability and absorption factor expressions are concerned, is that they are static and stratified. Therefore it is necessary to find a stratified model that best captures the radiating characteristics of the real solar atmosphere. For example, the exponential model is more effective than the VAL model in describing cross-limb flux ratio variations despite its empirical nature. This is because it takes account of the extension of the transition region into the corona due to spicule-like structures. 
However, the solar atmosphere is not simply stratified, nor is it static. A question therefore presents itself as to how structure affects the absorption characteristics in a plasma and the quantities derived thus far.

Absorption at a point is dependent upon the radiation field at that point. This radiation field is determined by the number of emitters in the plasma and the probability that photons emitted from them will reach the point in question. This probability is dependent upon the number of absorbers along the line of sight from the emitter to the absorber. Consider two plasmas of the same total number of emitters and absorbers, one stratified and one non-stratified. Absorption will be greater in the stratified plasma than in the non-stratified one since in the latter, the particles will appear more overlapped along each line of sight. This may be shown mathematically as follows: consider the absorption factor at layer centre. If the layer extends from $x=-D / 2$ to $x=D / 2$ then this is given by

$\Lambda\left(\tau_{0}, 0\right)=1-\frac{N_{l}(0)}{N_{u}(0)} \frac{\omega_{u}}{\omega_{l}} \frac{c^{2}}{2 \nu_{0}^{2}} \int I_{\nu} \phi_{\nu} \mathrm{d} \nu$

where

$I_{\nu}=\frac{1}{4 \pi} \iint_{V} \int \frac{j_{\nu}(\boldsymbol{r})}{r^{2}} e^{-\tau_{\nu}(\boldsymbol{r})} \mathrm{d} V$.

If it is written that

$\tau_{\nu}(\boldsymbol{r})=\alpha_{\nu} \int_{\boldsymbol{r} \rightarrow 0} N_{l}\left(\boldsymbol{r}^{\prime}\right) \mathrm{d} r^{\prime}$

and

$j_{\nu}(\boldsymbol{r})=\beta_{\nu} N_{u}(\boldsymbol{r})$

where

$\alpha_{\nu}=\frac{1}{c} h \nu B_{l \rightarrow u} \phi(\nu)$

and

$\beta_{\nu}=\frac{1}{4 \pi} A_{u \rightarrow l} \phi(\nu)$

then for a semi-infinite slab

$$
\begin{aligned}
I_{\nu} & =\frac{\beta_{\nu}}{4 \pi} \int_{-\pi}^{\pi} \int_{-\pi / 2}^{\pi / 2} \int_{0}^{D / 2 \cos \theta} N_{u}(\boldsymbol{r}) \\
& \times \exp \left\{-\alpha_{\nu} \int_{0}^{r} N_{l}\left(\boldsymbol{r}^{\prime}\right) \mathrm{d} r^{\prime}\right\}|\sin \theta| \mathrm{d} r \mathrm{~d} \theta \mathrm{d} \phi .
\end{aligned}
$$

It is useful to define $\bar{N}_{l}(\theta, \phi)$ and $\bar{N}_{u}(\theta, \phi)$ as

$$
\begin{aligned}
\bar{N}_{l}(\theta, \phi) & =\frac{\int_{0}^{D / 2 \cos \theta} N_{l}(\boldsymbol{r}) \mathrm{d} r}{D / 2 \cos \theta} \\
\bar{N}_{u}(\theta, \phi) & =\frac{\int_{0}^{D / 2 \cos \theta} N_{u}(\boldsymbol{r}) \mathrm{d} r}{D / 2 \cos \theta}
\end{aligned}
$$

and then to define $s$ as

$$
s=\frac{\int_{0}^{r} N_{l}\left(\boldsymbol{r}^{\prime}\right) \mathrm{d} r^{\prime}}{\bar{N}_{l}(\theta, \phi)}
$$

$$
\begin{aligned}
\Rightarrow \frac{\mathrm{d} s}{\mathrm{~d} r} & =\frac{N_{l}(\boldsymbol{r})}{\bar{N}_{l}(\theta, \phi)} \\
\Rightarrow I_{\nu} & =\frac{\beta_{\nu}}{4 \pi} \int_{-\pi}^{\pi} \int_{-\pi / 2}^{\pi / 2} \int_{0}^{D / 2 \cos \theta} \frac{N_{u}(\boldsymbol{r})}{N_{l}(\boldsymbol{r})} \bar{N}_{l}(\theta, \phi) \\
& \times e^{-\alpha_{\nu} \bar{N}_{l}(\theta, \phi) s}|\sin \theta| \mathrm{d} s \mathrm{~d} \theta \mathrm{d} \phi
\end{aligned}
$$

Now consider the regime where the source function is approximately constant. Then

$\frac{N_{u}(\boldsymbol{r})}{N_{l}(\boldsymbol{r})}=\frac{\bar{N}_{u}(\theta, \phi)}{\bar{N}_{l}(\theta, \phi)}$

and so

$$
\begin{aligned}
I_{\nu} & =\frac{\beta_{\nu}}{4 \pi} \int_{-\pi}^{\pi} \int_{-\pi / 2}^{\pi / 2} \int_{0}^{D / 2 \cos \theta} \bar{N}_{u}(\theta, \phi) \\
& \times e^{-\alpha_{\nu} \bar{N}_{l}(\theta, \phi) s}|\sin \theta| \mathrm{d} s \mathrm{~d} \theta \mathrm{d} \phi \\
& =\frac{\beta_{\nu}}{4 \pi} \int_{-\pi}^{\pi} \int_{-\pi / 2}^{\pi / 2} \frac{\bar{N}_{u}(\theta, \phi)}{\bar{N}_{l}(\theta, \phi)} \frac{1}{\alpha_{\nu}}[1 \\
& \left.-e^{-\alpha_{\nu} \bar{N}_{l}(\theta, \phi) D / 2 \cos \theta}\right]|\sin \theta| \mathrm{d} \theta \mathrm{d} \phi \\
& =\frac{S_{\nu}}{4 \pi} \int_{-\pi}^{\pi} \int_{-\pi / 2}^{\pi / 2}\left[1-e^{-\alpha_{\nu} \bar{N}_{l}(\theta, \phi) D / 2 \cos \theta}\right]|\sin \theta| \mathrm{d} \theta \mathrm{d} \phi
\end{aligned}
$$

where $S_{\nu}$ is the source function. The question is now, how does structuring with respect to $\theta$ and $\phi$ influence the radiation field at a point? To answer this, consider the integral

$\mathcal{I}_{\nu, \theta}=\int_{-\pi}^{\pi} e^{-\alpha_{\nu} \bar{N}_{l}(\theta, \phi) D / 2 \cos \theta} \mathrm{d} \phi$.

Intuitively, it is expected that structuring a plasma will lead to a reduction in the radiation field. This was discussed above. If this is so then introducing structure with respect to the angle $\phi$ in Eq. (33) will lead to an increase in $\mathcal{I}_{\nu, \theta}$. This may be shown to be the case as follows: write

$\mathcal{I}_{\nu, \theta}=\int_{-\pi}^{\pi} e^{-a_{\nu, \theta}\left(\overline{\bar{N}}_{l, \theta}+\varepsilon(\phi)\right)} \mathrm{d} \phi$

where

$a_{\nu, \theta}=-\alpha_{\nu} \frac{D}{2 \cos \theta}$

$\overline{\bar{N}}_{l, \theta}=\frac{1}{2 \pi} \int_{-\pi}^{\pi} \bar{N}_{l}(\theta, \phi) \mathrm{d} \phi$

$\varepsilon(\phi)=\bar{N}_{l}(\theta, \phi)-\overline{\bar{N}}_{l, \theta}$.

Here $\bar{N}_{l}(\theta, \phi)$ is written as its average over $\phi$ - i.e. $\overline{\bar{N}}_{l, \theta}-$ plus a quantity, $\varepsilon(\phi)$, which describes the deviation of $\bar{N}_{l}(\theta, \phi)$ from the average. It follows that

$\int_{-\pi}^{\pi} \varepsilon(\phi) \mathrm{d} \phi=0$ 
Euler's equation for Eq. (34) subject to the condition specified in Eq. (38) is

$\frac{\partial}{\partial \varepsilon}\left(e^{-a_{\nu, \theta}\left(\overline{\bar{N}}_{l, \theta}+\varepsilon(\phi)\right)}+\lambda \varepsilon(\phi)\right)=0$

where $\lambda$ is a Lagrangian multiplier and is constant. This implies that

$$
\begin{aligned}
-a_{\nu, \theta} e^{-a_{\nu, \theta}\left(\overline{\bar{N}}_{l, \theta}+\varepsilon(\phi)\right)}+\lambda & =0 \\
\Rightarrow \varepsilon(\phi) & =\text { const. }=0 .
\end{aligned}
$$

Thus with respect to $\phi$, the stratified case is an extremal.

To determine whether or not it is a maximal or minimal solution, consider the following example:

$\varepsilon(\phi)=\left\{\begin{array}{rr}1, & -\pi \leq \phi<0 \\ -1, & 0 \leq \phi<\pi .\end{array}\right.$

Then

$$
\begin{aligned}
\mathcal{I}_{\nu, \theta} & =\pi e^{a\left(\overline{\bar{N}}_{l, \theta}-1\right)}+\pi e^{a\left(\overline{\bar{N}}_{l, \theta}+1\right)} \\
& =\pi e^{a \overline{\bar{N}}}\left(e^{-1}+e\right)>2 \pi e^{a \overline{\bar{N}}} .
\end{aligned}
$$

Thus $\varepsilon(\phi)=0$ represents a minimal of Eq. (33) and a maximal of Eq. (32). Therefore, with respect to $\phi$, the stratified case is a minimal of $\Lambda\left(\tau_{0}, 0\right)$. That it is a minimal of $\Lambda\left(\tau_{0}, h\right)$ for all $h$ follows from symmetry.

The dependence of $\Lambda\left(\tau_{0}, 0\right)$ on structure with respect to $\theta$ does not follow easily in the plane-parallel case and so is demonstrated here for a spherical plasma.

For a spherical plasma, of radius $R$, the intensity at its centre is given by

$$
\begin{aligned}
I_{\nu} & =\frac{\beta_{\nu}}{4 \pi} \int_{-\pi}^{\pi} \int_{0}^{\pi} \int_{0}^{R} N_{u}(\boldsymbol{r}) \\
& \times \exp \left\{-\alpha_{\nu} \int_{0}^{r} N_{l}\left(\boldsymbol{r}^{\prime}\right) \mathrm{d} r^{\prime}\right\}|\sin \theta| \mathrm{d} r \mathrm{~d} \theta \mathrm{d} \phi \\
& =\frac{S_{\nu}}{4 \pi} \int_{-\pi}^{\pi} \int_{0}^{\pi}\left[1-e^{-\alpha_{\nu} R \bar{N}_{l}(\theta, \phi)}\right] \sin \theta \mathrm{d} \theta .
\end{aligned}
$$

Consider the integral

$$
\begin{aligned}
\mathcal{I}_{\nu, \phi} & =\int_{0}^{\pi} e^{-\alpha_{\nu} \bar{N}_{l}(\theta, \phi) R} \sin \theta \mathrm{d} \theta \\
& =\int_{0}^{\pi} e^{-b_{\nu, \phi}\left(\overline{\bar{N}}_{l, \phi}+\eta(\theta)\right)} \sin \theta \mathrm{d} \theta
\end{aligned}
$$

where

$$
\begin{aligned}
b_{\nu, \phi} & =-\alpha_{\nu} R \\
\overline{\bar{N}}_{l, \phi} & =\int_{0}^{\pi} \bar{N}_{l}(\theta, \phi) \sin \theta \mathrm{d} \theta \\
\eta(\theta) & =\bar{N}_{l}(\theta, \phi)-\overline{\bar{N}}_{l, \phi} .
\end{aligned}
$$

Hence

$\int_{0}^{\pi} \eta(\theta) \sin \theta \mathrm{d} \theta=0$
Euler's equation for Eq. (44) subject to the condition specified in Eq. (48) is then

$$
\begin{aligned}
\frac{\partial}{\partial \eta}\left(e^{-b_{\nu, \phi}\left(\overline{\bar{N}}_{l, \phi}+\eta(\theta)\right)} \sin \theta+\lambda \eta(\theta) \sin \theta\right) & =0 \\
\Rightarrow-b_{\nu, \theta} e^{-b_{\nu, \theta}\left(\overline{\bar{N}}_{l, \phi}+\eta(\theta)\right)} \sin \theta+\lambda \sin \theta & =0 \\
\Rightarrow \eta(\theta) & =\text { const. }=0 .
\end{aligned}
$$

Thus with respect to $\theta$, the stratified case is an extremal. Moreover, as before, with respect to $\theta$, the stratified case represents a minimal of $\Lambda\left(\tau_{0}, 0\right)$.

\subsection{Discussion}

It follows from the above that it is possible to have two plasmas of the same apparent optical depth in terms of emission, but different optical depths from the perspective of absorption. This might be observable in disk centre spectra. Consider the intensity ratio of the 2-2 to the $1-2$ component of the $\mathrm{C}$ III $2 \mathrm{~s} 2 \mathrm{p}{ }^{3} \mathrm{P}-2 \mathrm{p}^{2}{ }^{3} \mathrm{P}$ multiplet $(\sim 1175 \AA)$. Since these two lines arise from a common upper level, this ratio is proportional to that of their $\bar{g}\left\{\tau_{0}\right\}$ values which are determined by their line of sight optical depths. From an observation of this ratio at disk centre the optical depths of these lines may be deduced and from them the optical depths of all the other lines of C III may also be calculated. Thus all the C III optical depths may be known from a single $\bar{g}\left\{\tau_{0}\right\}$ ratio. This is discussed in Paper I. Now consider the intensity ratio of the $2-1$ to the $1-2$ component of the same C III multiplet. These lines do not share an upper level but have almost identical optical depths. Thus this ratio is proportional to that of their upper level population densities which is determined by their $\overline{\bar{g}}\left\{\tau_{0} / 2\right\}$ values $^{2}$. These in turn are dependent specifically upon optical depths at disk centre and thus the optical depths of all the $\mathrm{C}$ III may be deduced from the $I(2-1) / I(1-2)$ intensity ratio. If the plasma is stratified then the two sets of optical depth should be in agreement. If it is not then those inferred from the latter ratio will be lower than those of the former.

This reasoning follows from consideration of two plasmas of the same total number of particles. If, on the other hand, a plasma is de-homogenised by the removal rather than re-organising of particles - that is, so that the two plasmas are not of the same total particle number - then the same conclusion results. Removing plasma so as to leave spicule like structures, for example, means that at disk centre the optical depths are the same but the degree of absorption is reduced.

The latter perspective is more useful when considering spicule-like structures (i.e. radial structures) at disk centre. The former perspective (re-organisation rather than removal) is more useful when considering such structures at the limb.

\footnotetext{
${ }^{2} \overline{\bar{g}}\left\{\tau_{0} / 2\right\} \equiv \mathcal{G}\left(\tau_{0}, D / 2\right)$ in a constant density model (see Paper I)
} 


\section{Models with flow}

Plasma flow introduces a dependence of the absorption and emission profiles on position. In the zero-flow case the absorption and emission profiles share the same centroid location and so when flow is introduced it can only act so as to displace one profile with respect to the other. Only moderate opacities, where the source function is close to constant, are considered here. In this regime, and in the absence of line blending, opacity serves at most to influence line profiles by way of flattening their peaks. In more severely thick circumstances partial frequency redistribution, which leads to a dependence of the source function upon frequency, can lead to self-reversal of spectral lines. Thus for the optical depth regime considered here, if there is no line blending the only effect of this displacement is to reduce the degree of absorption. Thus the zero-flow, stratified case represents a minimal of $\Lambda\left(\tau_{0}, h\right)$. This may be illustrated as follows: consider the intensity, $I_{r}$, at the point 0 due to emission along a ray path of length $R$. This is given by

$$
\begin{aligned}
I_{r} & \sim \int_{0}^{R} \int_{0}^{\infty} \phi_{a}(\nu, 0) \phi_{e}(\nu, r) \\
& \times \exp \left\{-\int_{0}^{r} \kappa_{0}\left(r^{\prime}\right) \phi_{e}\left(\nu, r^{\prime}\right) \mathrm{d} r^{\prime}\right\} \mathrm{d} u \mathrm{~d} r
\end{aligned}
$$

where $\phi_{a}(\nu, r)$ and $\phi_{e}(\nu, r)$ respectively denote the absorption and emission profiles at the point $r$. For Gaussian profiles Eq. (50) may be written as

$$
\begin{aligned}
I_{r} & \sim \int_{0}^{R} \int_{-\infty}^{\infty} e^{-u^{2}} e^{-(u+\eta(r))^{2}} \\
& \times \exp \left\{-\int_{0}^{r} \kappa_{0}\left(r^{\prime}\right) e^{-\left(u+\eta\left(r^{\prime}\right)\right)^{2}} \mathrm{~d} r^{\prime}\right\} \mathrm{d} u \mathrm{~d} r
\end{aligned}
$$

where $\eta(r)$ is some function describing the Doppler shift of the line profile due to the relative flow velocity at the point $r$. If $f\left(\kappa_{0}, \eta, r\right)$ is defined such that

$$
I_{r} \sim \int_{0}^{R} f\left(\kappa_{0}, \eta, r\right) \mathrm{d} r
$$

then for extremals with respect to $\eta$, Euler's equation is

$$
\begin{aligned}
\frac{\partial f}{\partial \eta}=0 & \Rightarrow \int_{-\infty}^{\infty}\left[e^{-u^{2}} e^{-(u+\eta(r))^{2}}(-2(u+\eta(r)))\right. \\
& \times \exp \left\{-\int_{0}^{r} \kappa_{0}\left(r^{\prime}\right) e^{-\left(u+\eta\left(r^{\prime}\right)\right)^{2}} \mathrm{~d} r^{\prime}\right\} \\
& +e^{-u^{2}} e^{-(u+\eta(r))^{2}} \\
& \times \exp \left\{-\int_{0}^{r} \kappa_{0}\left(r^{\prime}\right)+e^{-\left(u+\eta\left(r^{\prime}\right)\right)^{2}} \mathrm{~d} r^{\prime}\right\} \\
& \left.\times \int_{0}^{r} \kappa_{0}\left(r^{\prime}\right) e^{-\left(u+\eta\left(r^{\prime}\right)\right)^{2}} 2\left(u+\eta\left(r^{\prime}\right)\right) \mathrm{d} r^{\prime}\right] \mathrm{d} u=0
\end{aligned}
$$

This is satisfied if $\eta(r)=$ const. (i.e. $\eta(r)=0$ ) for all values of $r$ and thus this condition specifies an extremal.
To determine whether or not it is a maximal or minimal solution, consider the case where at all points along the ray the plasma is stationary except at the point of absorption $(r=0)$ where there is flow parallel to the ray. In this case the line profile due to emission along the ray is displaced in frequency space from the absorption profile at $r=0$. The convolution of the two inevitably results in a decrease in absorption compared to the zero-flow case. Thus when there is no line blending the zero-flow case represents a minimal of the absorption factor.

In the case of line blending the situation becomes more complex. Blending itself leads to a decrease in the absorption factor and so since flow will contribute to the extent of blending, a decrease in the absorption factor results. Thus when there is blending, the zero-flow case does not necessarily represent a minimal.

In general flow and blending ought to be treated selfconsistently but if the main influence of flow is to broaden emission profiles then flow may be accounted for within the blending formulation. In this event flow is included implicitly. This has in fact already been the case in the analyses presented so far since spectral lines emanating from the transition region have Gaussian profiles broadened beyond their thermal widths due to non-thermal velocities (Spadaro et al. 1996). Figure 9 shows observed line profiles for the $\mathrm{C}$ II $2 \mathrm{~s}^{2} 2 \mathrm{p}^{2} \mathrm{P}_{3 / 2}-2 \mathrm{~s} 2 \mathrm{p}^{2}{ }^{2} \mathrm{P}_{1 / 2}$ line at $904.481 \AA$ (a) and the $\mathrm{C}$ III $2 \mathrm{~s} 2 \mathrm{p}^{3} \mathrm{P}_{2}-2 \mathrm{p}^{23} \mathrm{P}_{1}$ line at $1176.370 \AA$ (b). These lines are both unblended and neither show significant deviation from Gaussian.

\subsection{The validity of $\bar{g}\left\{\tau_{0}\right\}$ in the case of a non-stratified atmosphere with flow}

The escape probabilty approach hinges on the separability of the effects of opacity upon the population structure and on emergent fluxes. This separability de-couples and linearises the equations of radiative transfer and statistical balance. In a stratified, static atmosphere an optical depth regime exists whereby this separability is possible and where opacity effects are significant and observable. This regime is defined according to the degree of absorption within the plasma and the extent of the modification to the upper level population density distribution due to opacity which are characterised by the absorption factor, $\Lambda\left(\tau_{0}, h\right)$. Following this the influence of structure and flow upon the extent of this optical depth regime must be addressed. Both structure and flow serve to diminish the degree of absorption as compared with the stratified, static case, except when there is blending where flow can enhance the blending effects and thus lead to an increase in the degree of absorption. Providing that plasma flow influences emission profiles by way of broadening and does not distort their shape, then flow may be accounted for within blended lines as well as unblended ones. It is therefore appropriate to consider the stratified, static atmosphere in determining the region of validity of the $\bar{g}\left\{\tau_{0}\right\}$ quantity as structure and flow will not further restrict this region. 

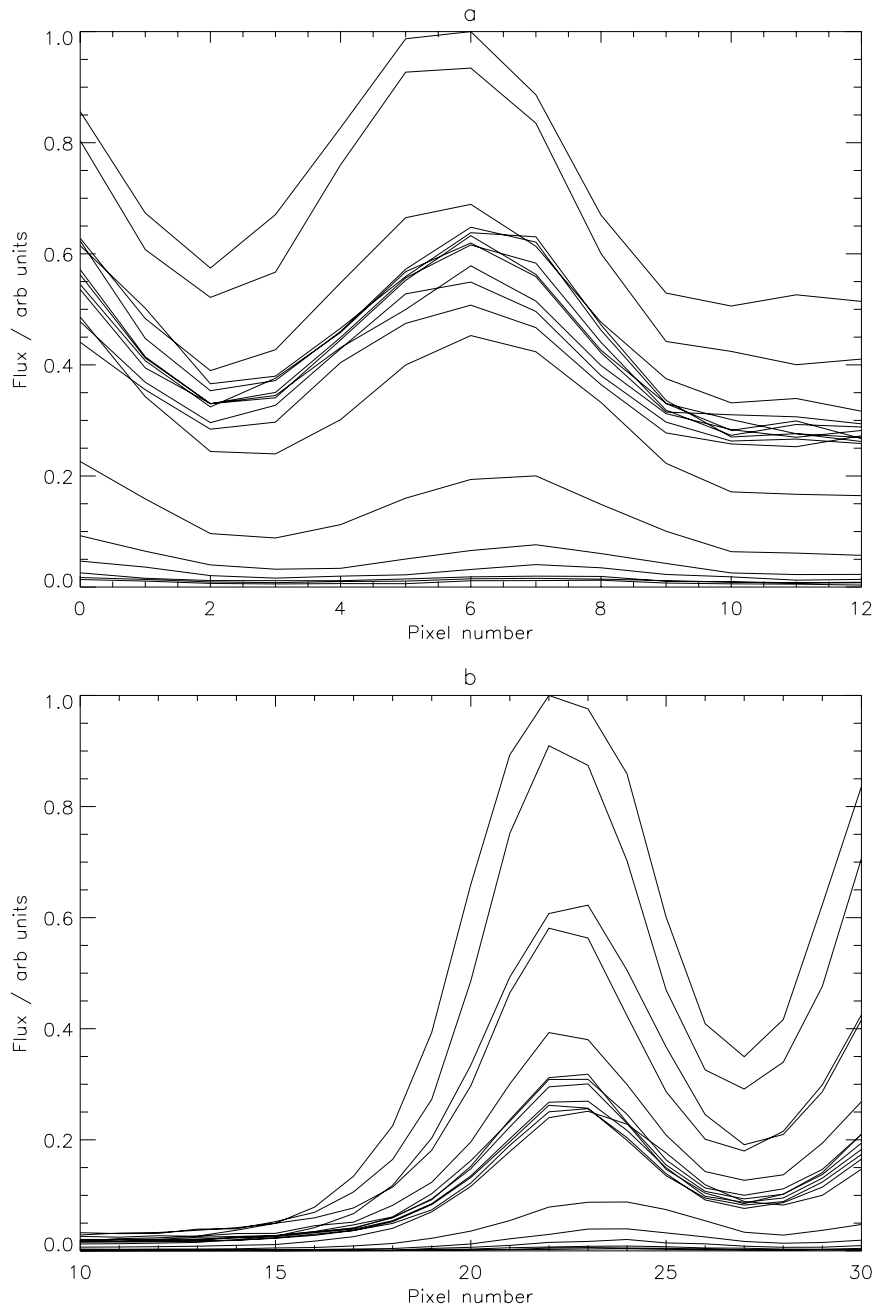

Fig. 9. Observed line profiles for a) the $\mathrm{C}$ II $2 \mathrm{~s}^{2} 2 \mathrm{p}^{2} \mathrm{P}_{3 / 2^{-}}$ $2 \mathrm{~s} 2 \mathrm{p}^{2}{ }^{2} \mathrm{P}_{1 / 2}$ line at $904.481 \AA$ and $\mathbf{b}$ ) the $\mathrm{C}$ III $2 \mathrm{~s} 2 \mathrm{p}{ }^{3} \mathrm{P}_{2}-2 \mathrm{p}^{23} \mathrm{P}_{1}$ line at $1176.370 \AA$. Profiles are shown for every pointing position. Although Doppler shifts are present (see Paper I), no significant asymmetries are evident. Therefore velocity fields lead only to line broadening and need not be explicitly accounted for in the escape probability/absorption factor analysis.

\section{Spatially varying source function}

Consider the specific cases of C II and C III. It was shown in Paper I that the maximum disk centre optical depths of lines of these two ions are $\sim 1.8$ and $\sim 0.16$ respectively. These values are within the regime of validity of $\bar{g}^{(i)}\left\{\tau_{0}\right\}$. Yet it was shown in Paper II that stratified models, in conjunction with $\bar{g}^{(i)}\left\{\tau_{0}\right\}$, are unable to accurately describe the $\mathrm{C}$ II and $\mathrm{C}$ III spectral characteristics observed by SOHO-SUMER in the vicinity of the solar limb even when the effects of instrumentally scattered light are included.

Following Eq. (7), Fig. 10 shows observed fluxes for the $\mathrm{C}$ III $2 \mathrm{~s} 2 \mathrm{p}{ }^{3} \mathrm{P}_{2}-2 \mathrm{p}^{2}{ }^{3} \mathrm{P}_{2}$ line at $1175.711 \AA$ with the function $\tau_{0} \bar{g}^{(i)}\left\{\tau_{0}\right\}$ overlaid. Here the optical depth at each point is not based on any model, stratified or otherwise. Rather the optical depths are those extracted from the observed ratios (see Table 1). The predicted fluxes are scaled to match the observed value at 946.46 arcsec.

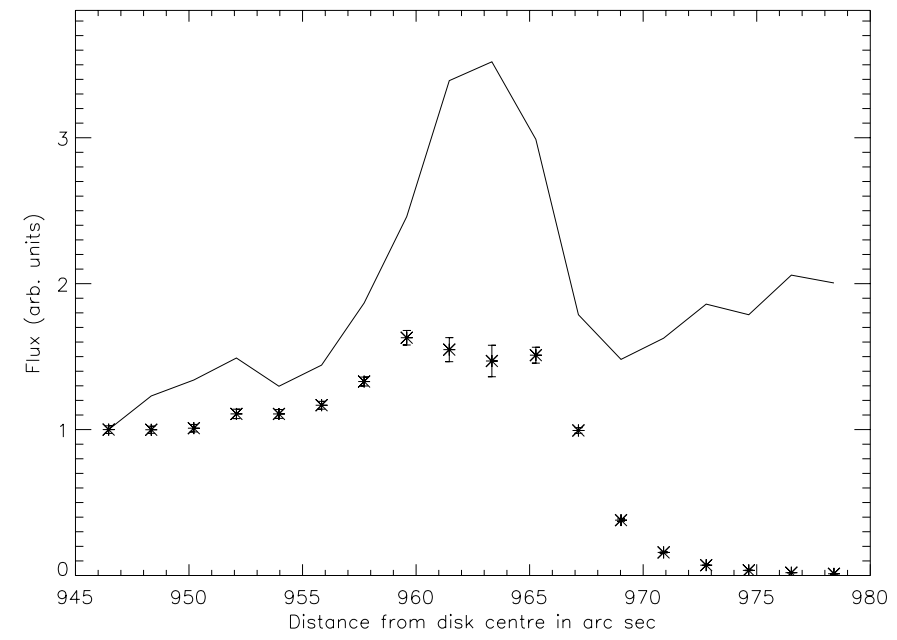

Fig. 10. Observed fluxes (*'s) for the C III $2 \mathrm{~s} 2 \mathrm{p}{ }^{3} \mathrm{P}_{2}-$ $2 \mathrm{p}^{2}{ }^{3} \mathrm{P}_{2}$ line at $1175.711 \AA$. Overlaid is the function $\tau_{0} \bar{g}^{(i)}\left\{\tau_{0}\right\}$ (solid curve) where the optical depths, $\tau_{0}$, for each position are taken from Table 1 . The predicted fluxes are scaled to match the observed ones at 946.46 arcsec.

The discrepancy is marked and denotes a failure in the escape probability model. Clearly at least one assumption underpinning the theory is invalid.

It has been shown that plasma flow and spectral line blending, which lead to a dependence of the source function on frequency, are, with respect to the $\mathrm{C}$ II and $\mathrm{C}$ III data presented here, either negligible or manageable within the escape probability framework. However, photon scattering can also lead to a dependence of the source function on frequency through partial frequency redistribution. Following Hubeny \& Lites (1995), although multiple scattering in the wings of the $\mathrm{L} \alpha$ line of hydrogen can lead to significant changes in the flux of that line which in turn can affect the charge balance and gas pressure through modification of the ionisation balance of hydrogen, the effects of partial frequency redistribution on line fluxes of other lines are not typically very large and so complete frequency redistribution is usually a good approximation. This is confirmed by Fig. 9 which shows no significant deviation from Gaussian profiles.

It has been shown that the modification to the source function due to opacity is not significant for either the C II or C III. However, as discussed above, there is a spatial variation of the source function due to the variation of electron density and temperature through the emitting layer. This variation was overlooked and the assumption was made that each emitting layer may be represented by a single $\left(T_{\mathrm{e}}, N_{\mathrm{e}}\right)$ pair. This assumption allowed for the modification to the source function purely due to opacity or alternatively, scattering into the line of sight - to be considered. It is conceivable that this variation is partially or wholly responsible for the breakdown of the $\tau_{0} \bar{g}^{(i)}\left\{\tau_{0}\right\}$ expression evident in Fig. 10. 
Consider the intensity from a layer of variable source function. Such emission is given by

$I \sim \int_{0}^{L} S(s) g\left(\alpha_{0} \int_{0}^{s} N_{l}\left(s^{\prime}\right) \mathrm{d} s^{\prime}\right) \mathrm{d} s$.

Here it is assumed that the source function, $S(s)$, is independent of frequency. Putting

$$
\begin{aligned}
t(s) & =\alpha_{0} \int_{0}^{s} N_{l}\left(s^{\prime}\right) \mathrm{d} s^{\prime} \\
\Rightarrow \frac{\mathrm{d} t}{\mathrm{~d} s} & =\alpha_{0} N_{l}(s)
\end{aligned}
$$

leads to

$$
\begin{aligned}
I & \sim \int_{0}^{L} S(s(t)) g(t) \mathrm{d} t \\
\Rightarrow I & \sim \bar{N}_{l} \bar{g}\left\{\tau_{0}\right\} L f_{\operatorname{los}}\left\{\tau_{0}\right\}
\end{aligned}
$$

where

$f_{\operatorname{los}}\left\{\tau_{0}\right\}=\int_{0}^{L} \frac{\tilde{N}_{u}(t)}{\tilde{N}_{l}(t)} \frac{g(t)}{\bar{g}\left\{\tau_{0}\right\}} \mathrm{d} t$

$f_{\text {los }}\left\{\tau_{0}\right\}$ is in general line of sight and optical depth dependent. The validity of using Eq. (5) to model branching ratios and limb-brightening curves is determined by the nature of the dependence of $f_{\operatorname{los}}\left\{\tau_{0}\right\}$ on $\tau_{0}$ and line of sight.

Equation (56) may be written as

$$
I \sim \tilde{N}_{l} \bar{g}\left\{\tau_{0}\right\} L
$$

where $\tilde{N}_{l}=\bar{N}_{l} f_{\text {los }}\left\{\tau_{0}\right\}$ is some representative upper level population density and is a function of optical depth and line of sight. This extends to blended lines if $\bar{g}^{(i)}\left\{\tau_{0}\right\}$ replaces $\bar{g}\left\{\tau_{0}\right\}$ in Eq. (56). The optical depth diagnostic described in Paper I depends upon the cancellation of the density terms in Eq. (5). The fact that two lines in an intensity ratio arise from a common upper level is no longer a guarantee that this cancellation occurs. There is therefore a key issue relating to the nature of the dependence of $f_{\text {los }}\left\{\tau_{0}\right\}$ on line of sight and optical depth.

Within the picture of thin sheaths around extended spicule-like structures (Mariska et al. 1978), the line of sight optical depth is principally determined by the number of structures intersected by that line of sight. Providing the conditions within each sheath are similar, the dependence of $f_{\text {los }}\left\{\tau_{0}\right\}$ on line of sight will be weak. The optical depth is smaller on-disk than at the limb for example the model based optical depth at disk centre for the $\mathrm{C}$ III $2 \mathrm{~s} 2 \mathrm{p}^{3} \mathrm{P}_{2}-2 \mathrm{p}^{2}{ }^{3} \mathrm{P}_{2}$ line at $1175.711 \AA$ is 0.16 (see Paper I) which compares with observationally deduced values of 0.61 and 1.40 at 946.46 and 967.15 arcsec respectively. Thus it may be deduced that for $\mathrm{C}$ II and C III optical depth of a single sheath is small and that the number of structures intersected by each line of sight in the vicinity of the limb is large.

If the variation of the source function with respect to space is responsible for the deviation of observed and predicted fluxes in Fig. 10, a correlation between this deviation and optical depth is expected. This would manifest itself at each raster position in a line-to-line variation of the deviation.

Another potential source for the deviation of predicted from observed fluxes is atmospheric structure. In Sect. 5 structure was discussed and it was demonstrated that it leads to a reduction in the degree of absorption within a plasma. Therefore, if the validity of $\bar{g}^{(i)}\left\{\tau_{0}\right\}$ is confirmed by consideration of the absorption factor within an appropriate stratified atmosphere model, then the presence of structure will strengthen rather than threaten this validity. Hence, from the perspective of the modification to the population structure (or scattering into the line of sight), structure does not influence the validity of the $\bar{g}^{(i)}\left\{\tau_{0}\right\}$ expression. Moreover, it was demonstrated in paper II that if it is assumed that the source function is constant in space, then the $\bar{g}^{(i)}\left\{\tau_{0}\right\}$ expression is independent of the nature of the variation of density along the line of sight. So structure along the line of sight is not responsible for the discrepancy in Fig. 10. However, structure will influence the fluxes if the observed structures are smaller in one or more dimension than the resolution limit of the instrument - i.e. if the observed structures are unresolved. If this is so then the term $f_{\operatorname{los}}\left\{\tau_{0}\right\}$ may be interpreted as an area filling factor. This is a purely geometric quantity and is therefore independent of optical depth.

If structure is responsible in this way for the discrepancy in Fig. 10, then in contrast to the case of the source function variation being responsible, a constancy of $f_{\text {los }}\left\{\tau_{0}\right\}$ with respect to $\tau_{0}$ - i.e. a constancy line-to-line - is expected.

Shown in Fig. 11 are observed fluxes versus raster position for each component of the $\mathrm{C}$ III $2 \mathrm{~s} 2 \mathrm{p}{ }^{3} \mathrm{P}-2 \mathrm{p}^{2}{ }^{3} \mathrm{P}$ multiplet with plots of $f_{\operatorname{los}}\left\{\tau_{0}\right\} \tau_{0} \bar{g}^{(i)}\left\{\tau_{0}\right\}$ overlaid. As in Fig. 10, the optical depths are those listed in Table 1. The factors $f_{\text {los }}\left\{\tau_{0}\right\}$ are determined such that the observed and predicted fluxes in Fig. 10 match exactly and so for each raster position there is only one scaling factor for all the lines of the multiplet. It can be seen from this figure that the single set of factors $f_{\text {los }}\left\{\tau_{0}\right\}$ is sufficient to match the predicted intensities to the observed ones almost exactly for each line with the exception of the 1-1 and 1-0 components. Both these are blended and the discrepancy between predicted and observed values is most significant in the vicinity of the limb which corresponds to the region of most significant optical thickness and thus also the greatest degree of blending. Furthermore the discrepancy is most marked for the 1-1 line which is in turn the more significantly influenced by blending since it is the weaker of the two lines. These facts suggest that the discrepancies in the 1-1 and 1-0 components relate to the difficulty in resolving these lines and not due a weakness in the escape probability analysis. It is also true that the $1-1$ and 1-0 components are the most optically thin of the multiplet (see Table 8 of Paper I) which might suggest that the discrepancies in these lines are due to a dependence of $f_{\text {los }}\left\{\tau_{0}\right\}$ on optical depth. However, the $1-0$ component has a comparable optical depth to that of the $0-1$ component 

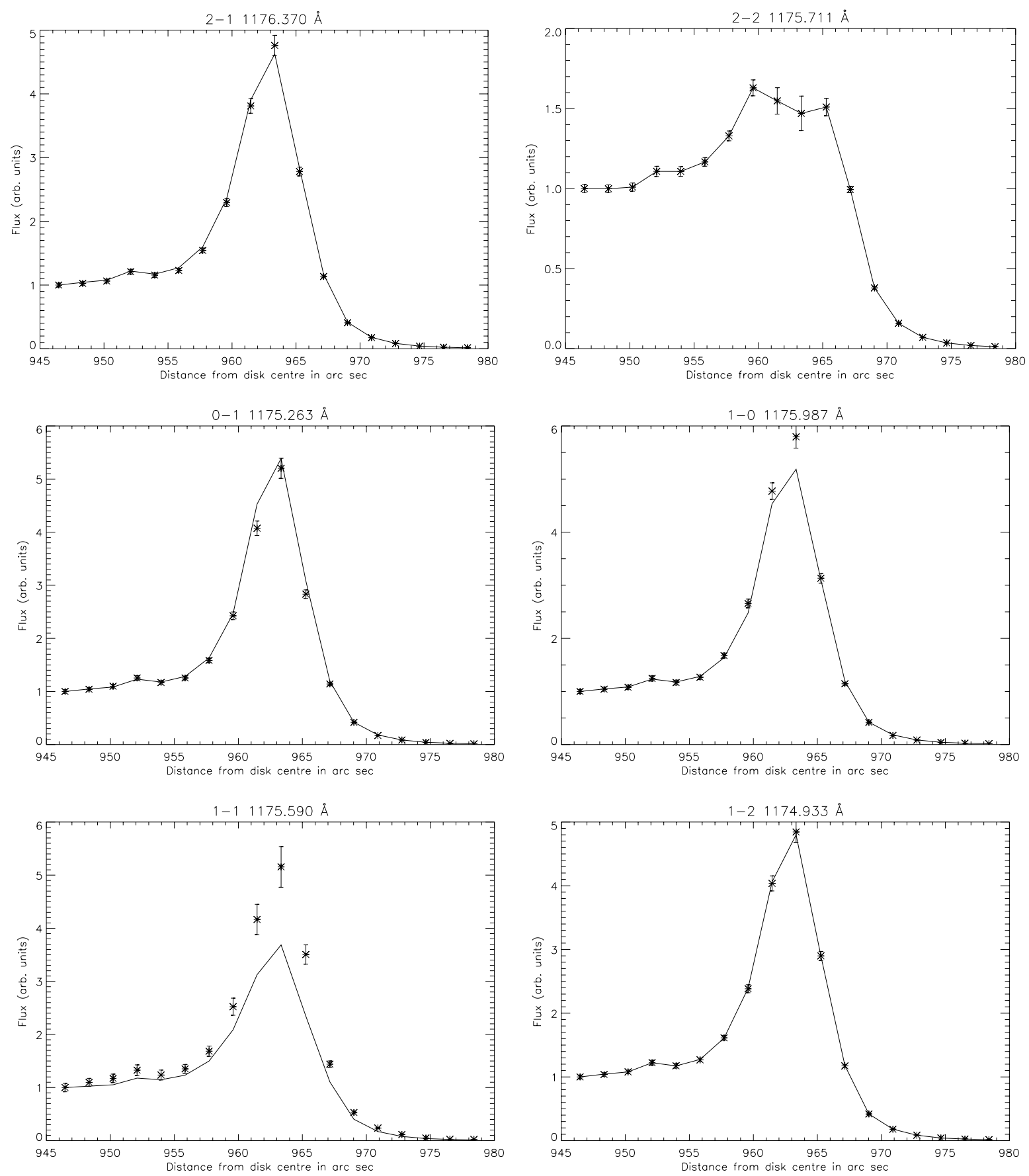

Fig. 11. Observed fluxes (*'s) versus raster position for each component of the C III $2 \mathrm{~s} 2 \mathrm{p}{ }^{3} \mathrm{P}-2 \mathrm{p}^{2}{ }^{3} \mathrm{P}$ multiplet. Overlaid are plots of $f_{\text {los }}\left\{\tau_{0}\right\} \tau_{0} \bar{g}^{(i)}\left\{\tau_{0}\right\}$ (solid curve) where the optical depths are those deduced from the observed branching ratios which are listed for the 2-2 component in Table 1. The factors $f_{\text {los }}\left\{\tau_{0}\right\}$ are determined such that the observed and predicted fluxes in Fig. 10 match exactly. The predicted fluxes are scaled to match the observed ones at 946.46 arcsec.

which shows good agreement between predicted and observed intensities.

Figure 12 shows the same as Fig. 11 but for the components of the $\mathrm{C}$ II $2 \mathrm{~s}^{2} 2 \mathrm{p}{ }^{2} \mathrm{P}-2 \mathrm{~s} 2 \mathrm{p}^{2}{ }^{2} \mathrm{P}$ multiplet. In these plots there is good agreement between model and observed fluxes for all the components with the only exception being the $1 / 2-1 / 2$ component which is not the most optically thin. In fact it is the closest in optical depth to the control line (i.e. the one that determines the $f_{\text {los }}\left\{\tau_{0}\right\}$ values).
It is, however, the most severely blended of all the lines of this multiplet.

Figure 13 shows observed fluxes versus raster position for both components of the C II $2 \mathrm{~s}^{2} 2 \mathrm{p}^{2} \mathrm{P}-2 \mathrm{~s} 2 \mathrm{p}^{2}{ }^{2} \mathrm{P}$ multiplet at $\sim 1036 \AA$ again with plots of $f_{\operatorname{los}}\left\{\tau_{0}\right\} \tau_{0} \bar{g}^{(i)}\left\{\tau_{0}\right\}$ overlaid. Here there is near perfect agreement between the two lines, neither of which are blended.

It follows then that $f_{\operatorname{los}}\left\{\tau_{0}\right\}$ is independent of optical depth, $\tau_{0}$, since the same set of factors, $f_{\text {los }}\left\{\tau_{0}\right\}$, are 

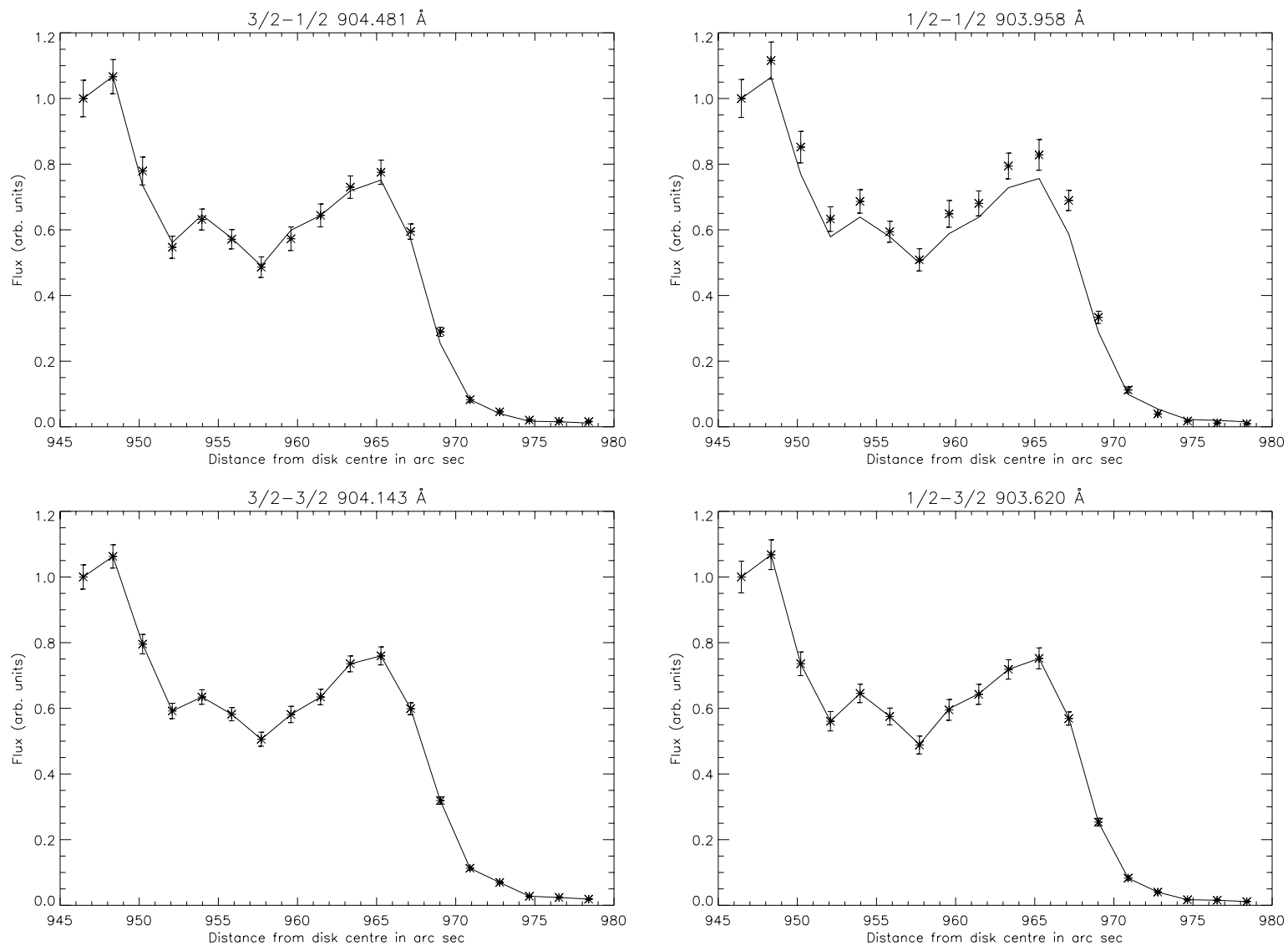

Fig. 12. Observed fluxes (*'s) versus raster position for each component of the C II $2 \mathrm{~s}^{2} 2 \mathrm{p}{ }^{2} \mathrm{P}-2 \mathrm{~s} 2 \mathrm{p}^{2}{ }^{2} \mathrm{P}$ multiplet. Overlaid are plots of $f_{\text {los }}\left\{\tau_{0}\right\} \tau_{0} \bar{g}^{(i)}\left\{\tau_{0}\right\}$ (solid curve) where the optical depths are those deduced from the observed branching ratios which are listed for the 3/2-3/2 in Table 3. The factors $f_{\text {los }}\left\{\tau_{0}\right\}$ are determined such that the observed and predicted fluxes for the 3/2-3/2 component match exactly. The predicted fluxes are scaled to match the observed ones at 946.46 arcsec.

effective for each line even though the optical depth varies line-to-line. Further confirmation of this follows from the findings of Paper II in which it was found that at the limb the stratified models, coupled with the escape probability techniques, overestimate both the emergent fluxes and the $\mathrm{C}$ III branching ratios at the limb. Furthermore, at heights above $\sim 970$ arcsec both the $\mathrm{C}$ II and $\mathrm{C}$ III emergent fluxes are underestimated whereas the branching ratios are overerestimated if the effects of instrumentally scattered light are neglected. It follows that at the limb the stratified model predicts column densities too great to match the emergent fluxes but too small to match the ratios. The inclusion of scattered light is not sufficient to remove this discrepancy. The discrepancy is removed, however, if the emitting structures in the vicinity of the limb are partially or wholly unresolved. Well beyond the limb the ratios indicate optical depths comparable with those on the disk despite the fact that the observed fluxes are significantly lower than those in this region.

This body of evidence demonstrates that the discrepancy in Fig. 10 is not due to the spatial variation of the source function but is due to structure. It follows that the neglect of the dependence of the source function on space is appropriate and the line of sight averaged escape probability is effective.

\section{Implications for transition region structure}

It has been concluded that $f_{\text {los }}\left\{\tau_{0}\right\}$ may be interpreted as a filling factor, which represents the area of the slit occuppied by emitting structures. Since it is independent of optical depth, it may be written as $f_{\text {los }}$. The deduced filling factors for the C II multiplets at 904 and $1036 \AA$ are shown in Figs. 14a and b respectively and those of the $\mathrm{C}$ III multiplet at $1775 \AA$ are shown in Fig. 15. In each case the factors are scaled to be unity at 946.46 arcsec. There is a general downward trend evident in Figs. 14a and 15 indicating a decrease in occupation of the slit by emitting structures with distance from disk centre. No such trend is evident in Fig. 14b. In each figure, however, strong variations are evident. This variability implies that the absolute filling factors are unity at no more than one pointing position and are thus unlikely be unity anywhere. This implies the presence of unresolved structures at each pointing position - i.e. on the disk, at the limb and beyond.

Of course, the observed fluxes are summed over the slit which has a length of $\sim 60$ arcsec. Thus the filling factors here correspond to the presence of spicule-like structures of diameter less than 60 arcsec. If at each pointing position the pixels are sorted according to their brightness, then the data may be split into regions associated with 

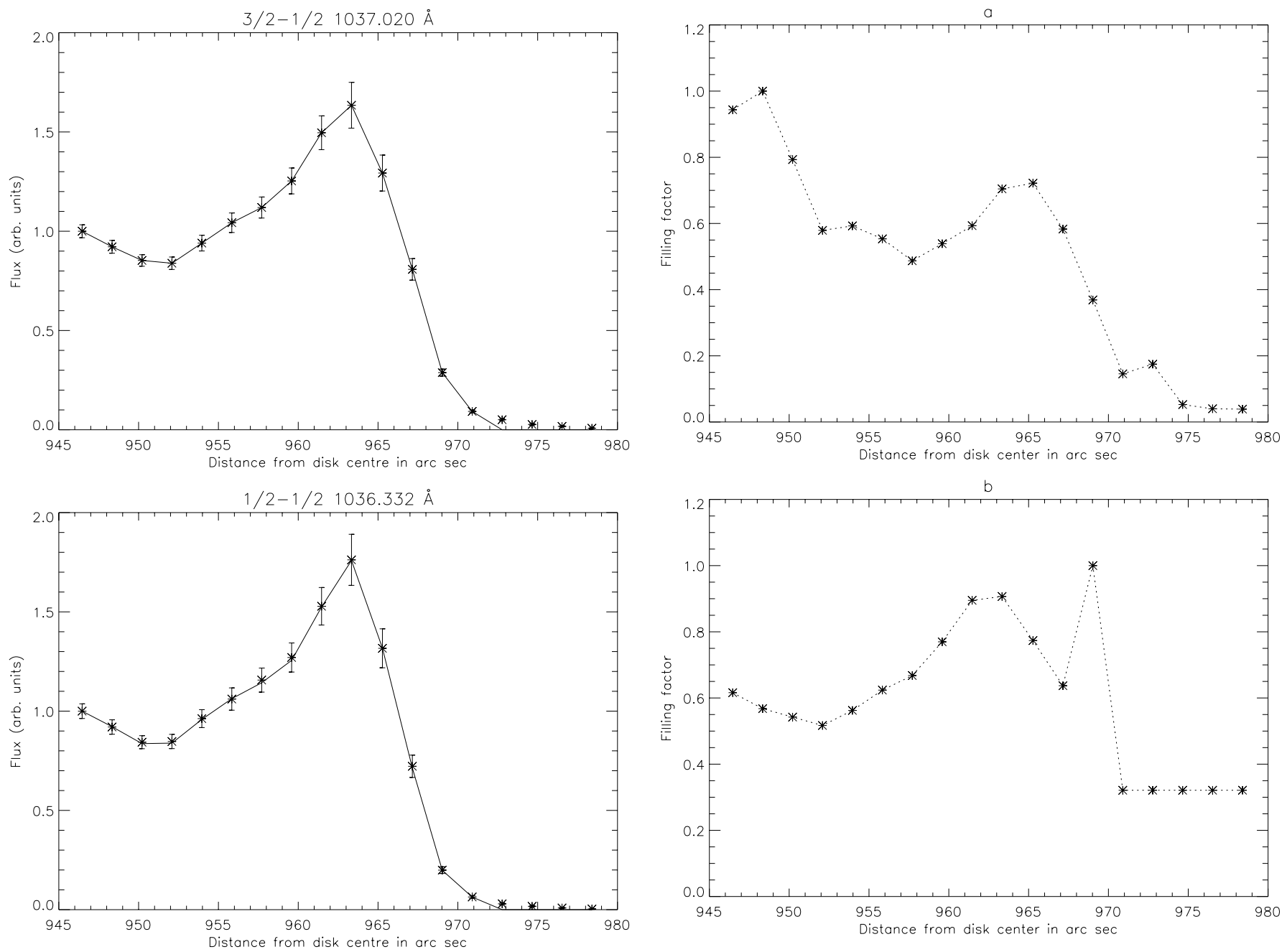

Fig. 13. Observed fluxes (*'s) versus raster position for each component of the $\mathrm{C}$ II $2 \mathrm{~s}^{2} 2 \mathrm{p}{ }^{2} \mathrm{P}-2 \mathrm{~s} 2 \mathrm{p}^{2}{ }^{2} \mathrm{P}$ multiplet. Overlaid are plots of $f_{\text {los }}\left\{\tau_{0}\right\} \tau_{0} \bar{g}^{(i)}\left\{\tau_{0}\right\}$ (solid curve) where the optical depths are those deduced from the observed branching ratios which are listed for the $3 / 2-1 / 2$ in Table 2 . The factors $f_{\text {los }}\left\{\tau_{0}\right\}$ are determined such that the observed and predicted fluxes for the $3 / 2-1 / 2$ component match exactly. The predicted fluxes are scaled to match the observed ones at 946.46 arcsec.

resolved structures and regions that are visibly in between the observable structures.

Figures $16 \mathrm{a}$ and $\mathrm{b}$ show observed and model fluxes of the $\mathrm{C}$ III $2 \mathrm{~s} 2 \mathrm{p}^{3} \mathrm{P}_{2}-2 \mathrm{p}^{2}{ }^{3} \mathrm{P}_{2}$ line at $1175.711 \AA$ for regions associated and not associated with observable spicule-like structure. For regions associated with structure the stratified model clearly fails as before. For the intra-spicular regions, however, the stratified model is much more effective. Figures $17 \mathrm{a}$ and $\mathrm{b}$, on the other hand, show the same observed fluxes this time in comparison with $\tau_{0} \bar{g}^{(i)}\left\{\tau_{0}\right\}$. In both cases there is a significant deviation between observed and predicted values, indicating the presence of unresolved structures even in the intra-spicular data.

Fig. 14. C II filling factors $\left(f_{\text {los }}\right)$ versus pointing position for lines of a) the $904 \AA$ multiplet and b) the $1036 \AA$ multiplet. In $\mathbf{b})$, the filling factor values for the four highest pointing positions are set to the value at 970.9 arcsec since no optical depths were deduced for these points (see Table 2).

\section{Conclusions}

The assumptions underpinning the simple escape probability and absorption factor techniques for describing the effects of opacity on emergent flux and population structure have been addressed. Spatially resolved calculations of the effects of scattering into the line of sight in a stratified atmosphere have been presented, including and excluding spectral line blending. These have led to the identification of a disk centre optical depth regime within which the line of sight averaged escape probability, $\bar{g}^{(i)}\left\{\tau_{0}\right\}$, is valid. In the unblended case the upper limit on the disk centre optical depth is $\sim 10$. This is identical to that found by Kastner (1999) based on an intensity/linewidth method to deduce optical depths from spectral observations using escape probabilities. Indirect effects on absorption result in the range of validity of the absorption factor $\mathcal{G}\left(\tau_{0}, x\right)$ being more restricted (e.g. $0 \leq$ $\tau_{0}<\sim 1$ in the unblended case). 


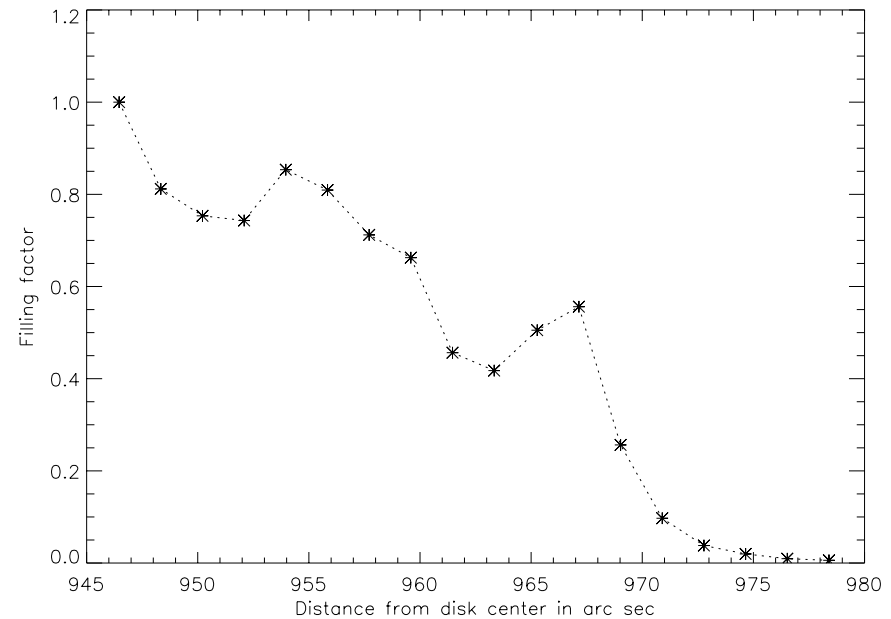

Fig. 15. C III $1775 \AA$ multiplet filling factors versus pointing position.
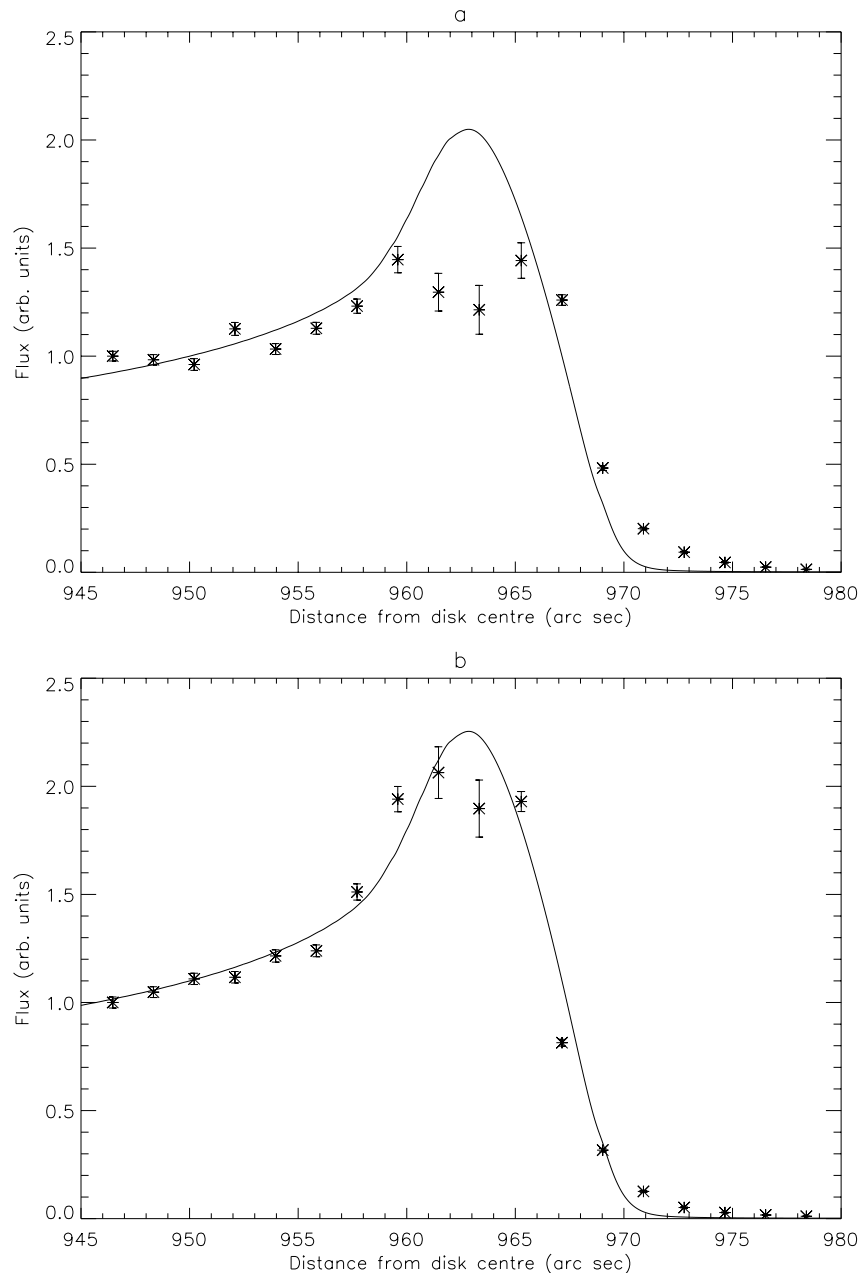

Fig. 16. Observed fluxes (*'s) of the C III $2 \mathrm{~s} 2 \mathrm{p}{ }^{3} \mathrm{P}_{2}-2 \mathrm{p}^{2}{ }^{3} \mathrm{P}_{2}$ line at $1175.711 \AA$ with predicted values based on an exponential density model overlaid, for a) regions associated with observable structure and $\mathbf{b}$ ) regions not associated with observable structure. The calculations include the effects of instrumentally scattered light.

It has been demonstrated that if the escape probability is valid for a stratified atmosphere then it is also
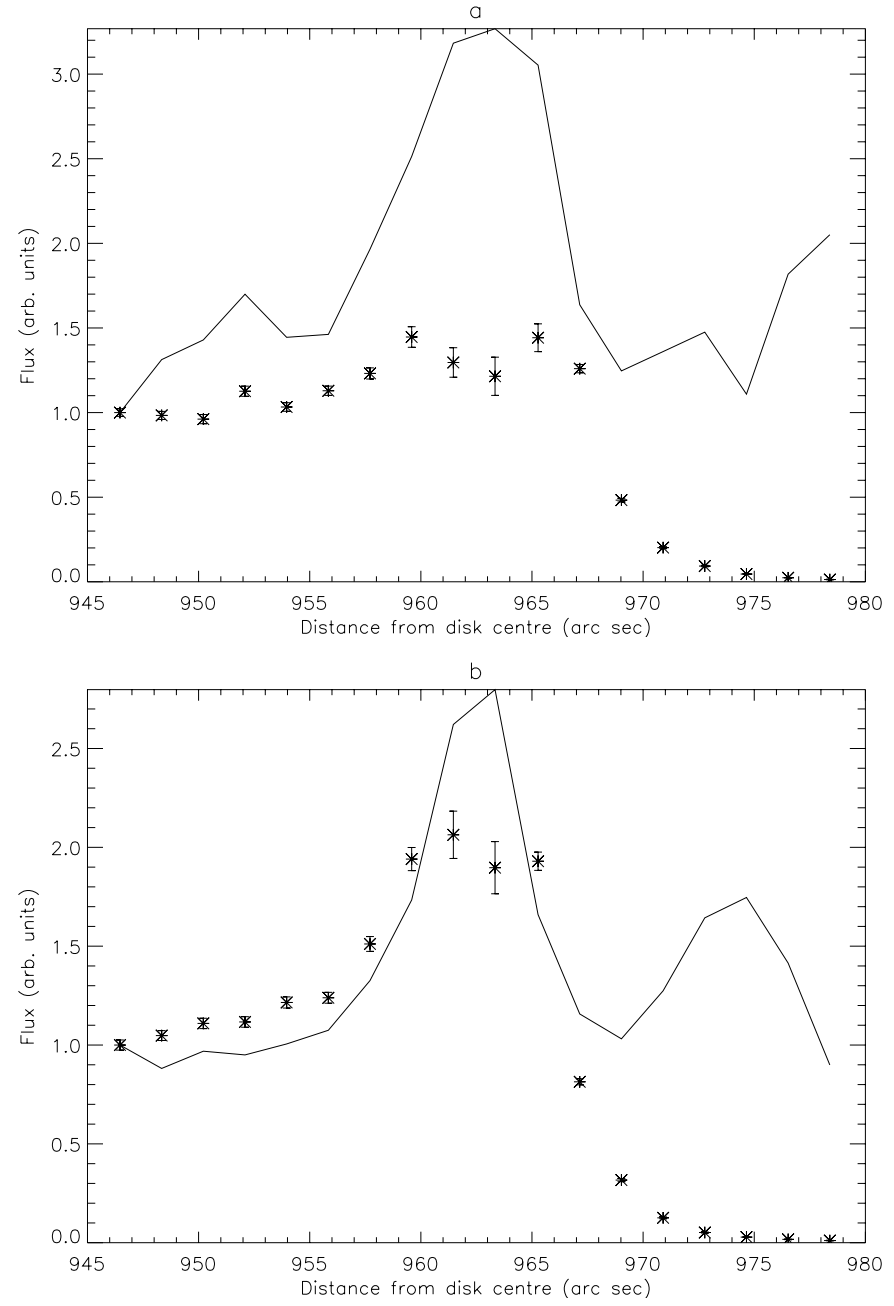

Fig. 17. Observed fluxes (*'s) of the C III $2 \mathrm{~s} 2 \mathrm{p}^{3} \mathrm{P}_{2}-2 \mathrm{p}^{2}{ }^{3} \mathrm{P}_{2}$ line at $1175.711 \AA$ with $\tau_{0} \bar{g}^{(i)}\left\{\tau_{0}\right\}$ overlaid, for a) regions associated with observable structure and $\mathbf{b}$ ) regions not associated with observable structure.

valid in the non-stratified case. This is because the influence of structure is to reduce the degree of absorption. Furthermore, if plasma flow serves purely to broaden the spectral lines and not distort them, then the escape probability is also valid in the dynamic atmosphere case.

It follows that the extraction of optical depths of spectral lines from observed branching ratios is reliable. Moreover, it has been found that in the C II and C III data from SUMER presented here and in Papers I and II of this series, the discrepency between the observed fluxes and $\tau_{0} \bar{g}^{(i)}\left\{\tau_{0}\right\}$, where the $\tau_{0}$ values are those extracted directly from the data, may be interpreted in terms of an area filling factor. This is indicative of the presence of spicule-like structures. Analysis of data separated into spicular and intra-spicular regions also yields filling factors which suggests the presence of structures that are observationally unresolved. 


\section{References}

Anzer, U., \& Heinzel, P. 1999, A\&A, 349, 974

Anzer, U., \& Heinzel, P. 2000, A\&A, 358, L75

Auer, L. H., \& Paletou, F. 1994, A\&A, 285, 675

Bhatia, A. K., \& Kastner, S. O. 1997, J. Quant. Spectrosc. Radiat. Trans., 58, 347

Bhatia, A. K., \& Kastner, S. O. 1999, ApJ, 516, 482

Brooks, D. H., Fischbacher, G. A., Fludra, A., et al. 2000, A\&A, 357, 697

Carlsson, M. 1986, Technical Report 33, Uppsala Astronomical Observatory

Code, A. D., \& Whitney, B. A. 1995, ApJ, 441, 400

Doyle, J. G., \& McWhirter, R. W. P. 1980, MNRAS, 193, 947

Doyle, J. G., Teriaca, L., \& Banerjee, D. 2000, A\&A, 356, 335

Fischbacher, G. A., Loch, S. D., \& Summers, H. P. 2000, A\&A, 357,767

Fontenla, J. M, Rovira, M., Vial, J.-C., et al. 1996, ApJ, 466, 496

Gontikakis, C., Vial, J.-C., \& Gouttebroze, P. 1997, A\&A, 325, 803

Gouttebroze, P., Vial, J.-C., \& Tsiropoula, G. 1986, A\&A, 154, 154

Heinzel, P. 1995, A\&A, 299, 563

Heinzel, P., Gouttebroze, P., \& Vial, J.-C. 1987, A\&A, 183, 351

Holstein, T. 1947, Phys. Rev., 72, 1212

Hubeny, I., \& Lites, B. W. 1995, ApJ, 455, 376

Hummer, D. G., \& Rybicki, G. B. 1982, ApJ, 263, 925
Irons, F. E. 1979, J. Quant. Spectrosc. Radiat. Transfer, 22, 1 Jordan, C. 1967, Sol. Phys., 2, 441

Kastner, S. O. 1999, Phys. Scr., 60, 381

Kastner, S. O., \& Bhatia, A. K. 1992, ApJ, 401, 416

Kastner, S. O., \& Kastner, R. E. 1990, J. Quant. Spectrosc. Radiat. Transfer, 44, 2, 275

Keenan, R. P., \& Kingston, A. B. 1986, MNRAS, 220, 493

Lanzafame, A. C. 1994, A\&A, 287, 792

Mariska, J. T., Feldman, U., \& Doschek, G. A. 1978, ApJ, 226, 698

McWhirter, R. W. P. 1965, in Plasma Diagnostic Techniques, ed. R. H. Huddlestone, \& D. H. Leonard (Academic Press, Inc.)

Mein, N., Mein, P., Heinzel, et al. 1996, A\&A, 309, 275

Olson, G. L., Auer, L. H., \& Buchler, J. R. 1986, J. Quant. Sectrosc. Radiat. Transfer, 35, 431

Orrall, F. Q., \& Schmahl, E. J. 1980, ApJ, 240, 908

Paletou, F. 1996, A\&A, 311, 708

Paletou, F., Vial, J.-C., \& Auer, L. H. 1993, A\&A, 274, 571

Spadaro, D., Lanza, A. F., \& Antiochos, S. K. 1996, ApJ, 462, 1011

Summers, H. P. 2001, The ADAS User Manual, Version 2.3, webpage http://adas.phys.strath.ac.uk (Glasgow: University of Strathclyde)

Vernazza, J. B., Avrett, B. H., \& Loeser, R. 1981, ApJS, 45, 635

Wood, K., \& Raymond, J. 2000, ApJ, 540, 563

Zheng, W., Davidsen, A. F., \& Kriss, G. A. 1998, ApJ, 115, 391 